\title{
An overview of multiscale molecular modelling and simulation of silica aerogels
}

\author{
Pedro Maximiano, Luísa Durães, and Pedro Simões* \\ CIEPQPF, Department of Chemical Engineering, University of Coimbra, Rua Silvio de \\ Lima, 3030-790 Coimbra, Portugal \\ E-mail: pnsim@eq.uc.pt \\ Phone: +351-239798732
}

\begin{abstract}
Molecular simulation has become an integral and invaluable part of Chemical Product Engineering, as it provides fundamental and indispensable insights for a rational product design. Silica aerogels are materials with exceptional properties and correspondingly broad applications whose study has benefited from this new paradigm. The complex physical/chemical phenomena involved in their synthesis are hard to explain with experimental data alone. This document reviews relevant atomistic studies regarding silica aerogels, highlighting their contributions to the understanding of the microscopic phenomena inherent to the sol-gel process. Quantum mechanics calculations have provided a framework for investigating the mechanisms, energetics and products of silica precursor hydrolysis and condensation reactions. Classical molecular dynamics simulations have proven to be suitable for modelling the nanoscale porous network structure of the aerogels, allowing the reliable estimation of structural, mechanical, thermal and surface properties. Coarse graining models have allowed the extension of these studies to the mesoscale.
\end{abstract}




\section{Introduction}

Molecular modelling and simulation (MM\&S) tools have now become a crucial option to get meaningful, often unique, data to supplement/complement and even drive forward experimental research on a wide range of areas, gaining traction in both academic and industrial domains ${ }^{1-4}$. This new paradigm was made possible in the last two decades as computer hardware has become exponentially more powerful and theoretical methods and algorithms have continuously increased in quality and efficiency. The now widespread use of this kind of rational in silico approaches in the chemical industry is undeniable, as they help fuel the modern era of Chemical Product Engineering that is focused towards the design of novel valuable products. ${ }^{5-8}$ MM\&S methods have the ability to explore physico-chemical and biological phenomena on a fundamental level, which is very hard to capture by experimental analysis alone as they often lack such level of detail. ${ }^{8}$

Silica aerogels, first introduced by Kistler in $1931^{9}$, are even today one of the most important and highly investigated nanostructured materials. They possess outstanding properties, such as very low thermal conductivity (finding wide applications in the aerospace industry), very low density, high porosity and high surface area (useful as catalytic supports, Cherenkov detectors and as adsorbents of organic and heavy metal pollutants in water and soils, among others), low dieletric constant (useful for a variety of electronic components), good optical properties, etc. ${ }^{10,11}$

Silica aerogels are synthesized via the sol-gel method, which comprises the following steps: hydrolysis, condensation, gelation, aging, gel washing, and gel drying. ${ }^{9,10,12}$ The starting point is a solution of precursor molecules (in a water/alcohol mixture), usually alkoxysilanes (see Figure 1). The use of alkoxysilanes is preferred as they allow easy control over the structure of the final material, and because they provide a versatile means of introducing functional moieties (either by having co-precursors in the initial mixture or by a later stage of surface modification). The first step is the hydrolysis of these molecules to produce reactive hydroxyl groups ${ }^{13}$ :

$$
\begin{gathered}
\equiv \mathrm{Si}-\mathrm{OR}+\mathrm{H}_{2} \mathrm{O} \underset{\text { Esterification }}{\stackrel{\text { Hydrolysis }}{\rightleftharpoons}} \equiv \mathrm{Si}-\mathrm{OH}+\mathrm{R}-\mathrm{OH} \\
\text { ACS Paragon Plys Environment }
\end{gathered}
$$


<smiles>CO[Si](OC)(OC)OC</smiles>

Tetramethylorthosilicate

(TMOS)<smiles>CCO[Si](OCC)(OCC)OCC</smiles>

Tetraethylorthosilicate (TEOS)<smiles>CO[Si](C)(OC)OC</smiles>

Methyltrimethoxysilane (MTMS)<smiles>C=C[Si](OC)(OC)OC</smiles>

Vinyltrimethoxysilane (VTMS)

Figure 1: Examples of alkoxysilane precursors commonly used in sol-gel synthesis of silica aerogels.

After being hydrolyzed, the precursor molecules react with one another by multiple condensation reactions creating siloxane bridges, and primary particles $(<1 \mathrm{~nm})$ are nucleated, which agglomerate into secondary particles (size of tens of nanometers), creating a $\operatorname{sol}^{13}$ :

$$
\begin{gathered}
\equiv \mathrm{Si}-\mathrm{OH}+\mathrm{HO}-\mathrm{Si} \equiv \frac{\text { Condensation }}{\underset{\text { Hydrolysis }}{\rightleftharpoons}} \equiv \mathrm{Si}-\mathrm{O}-\mathrm{Si} \equiv+\mathrm{H}_{2} \mathrm{O} \\
\equiv \mathrm{Si}-\mathrm{OR}+\mathrm{HO}-\mathrm{Si} \equiv \frac{\text { Condensation }}{\underset{\text { Alcoholysis }}{\rightleftharpoons}} \equiv \mathrm{Si}-\mathrm{O}-\mathrm{Si} \equiv+\mathrm{R}-\mathrm{OH}
\end{gathered}
$$

Because of the low positive partial charge of Si atoms in the silica alkoxysilane precursors $\left(\delta_{\mathrm{Si}}=+0.32\right)$, compared to other metal alkoxides ${ }^{9}$, hydrolysis and condensation reactions can be extremely slow at neutral $\mathrm{pH}$ and at ambient temperature. Therefore, it is common to catalyze both processes through the use of acids or bases. ${ }^{9,10}$ The hydrolysis and condensation reactions can be controlled separately by employing a two-step catalysis process: acid catalysis favors hydrolysis reactions, while a subsequent shift to basic conditions tends to favor condensation/polycondensation. ${ }^{9}$ The mechanisms of condensation and the resulting products change significantly for different $\mathrm{pH}$ conditions: acidic conditions lead to gels with linear chains, which are prone to break due to more favorable hydrolysis of siloxane bonds, while higher $\mathrm{pH}$ leads to a crosslinked gel matrix with branched chains. ${ }^{10}$ When the condensation process reaches the entire volume of the sol, gelation (by polycondensation) takes place as primary particles aggregate onto secondary particles, which then link to form a three-dimensional network, with a pearl necklace-like structure (see Figure 2).

Once the gel matrix has been obtained, an aging step is usually employed in order to strengthen it, which is accomplished by two simultaneous phenomena: Ostwald ripen- 


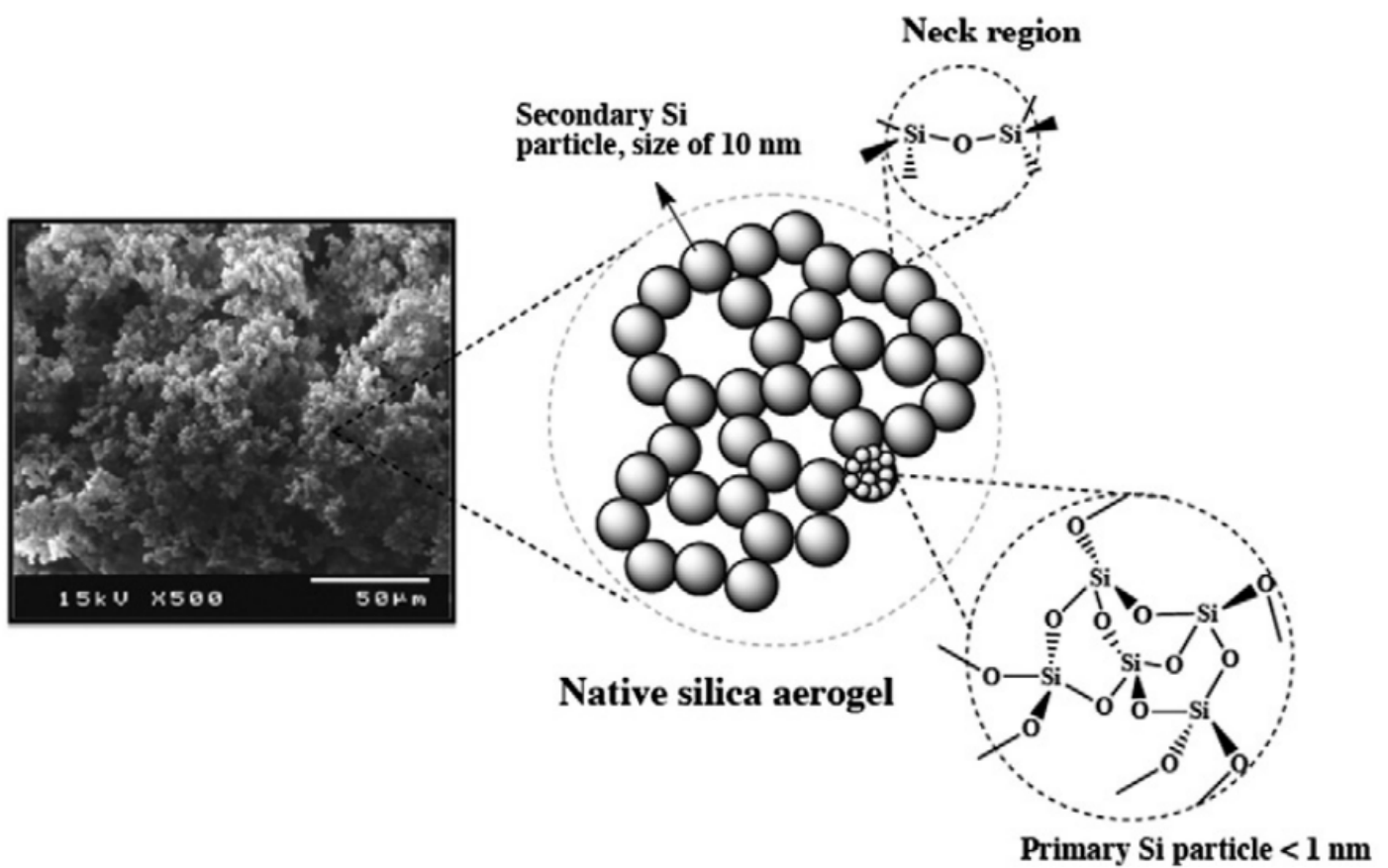

Figure 2: Schematic representation of the microscopic structure of a silica aerogel. Republished with permission of Royal Society of Chemistry, from Journal of materials chemistry A, Materials for energy and sustainability, "Advances in carbon nanostructure-silica aerogel composites: a review", Alyne Lamy-Mendes, Rui F. Silva and Luísa Durães, 6, 4, 2018, p. 1340-1369 ${ }^{14}$; permission conveyed through Copyright Clearance Center, Inc.

ing, in which small particles are dissolved and then reprecipitated onto larger particles; and neck growth, due to re-precipitation of dissolved silica onto the regions connecting secondary particles ("necks"). ${ }^{9,15}$ The aged gel can then be washed - which allows the removal of unconverted reactants and additives and, if needed, perform solvent exchange - and finally dried, in order to extract the entire liquid phase surrounding the gel matrix. Depending on the drying procedure used, very different materials can be obtained since phase changes in the solvent induce capillary forces that cause pore collapse and irreversible shrinkage of the network. ${ }^{9-11,15}$ According to Vareda et al. ${ }^{11}$, only the dried gels whose structure is not significantly impacted by the drying procedure can be considered an aerogel. As such, there are currently only two routes that yield silica aerogels: (a) supercritical drying (SCD) - either by removing the solvent in supercritical conditions (high temperature SCD) or extracting it with a supercritical fluid (low temperature SCD, using supercritical $\mathrm{CO}_{2}$ ); (b) ambient pressure drying (APD) combined with strategies to lower the surface tension of the solvent, such as solvent exchange, addition of surfactants and chemical modification of the gel surface. ${ }^{9-11,15}$ 
Due to its complexity, sol-gel chemistry is a prime example of how MM\&S approaches provide nanometric and sub-nanometric details unattainable solely by experimentation. For instance, NMR spectroscopy has the ability to determine which Si environments are present in the structures being formed during the sol-gel condensation reactions. They can be classified according to the $\mathrm{Q}^{n}$ notation, where $n$ is the number of $\mathrm{Si}$ atoms connected to the $\mathrm{Si}$ atom in question by $-\mathrm{O}$ - bridges (Figure 3). ${ }^{9,16}$ $\mathrm{Q}^{1}$ and $\mathrm{Q}^{2}$ are characteristic of linear species, while $\mathrm{Q}^{3}$ and $\mathrm{Q}^{4}$ are characteristic of branched forms. ${ }^{9,17}$ However, a NMR spectrum is the overall result of contributions from a myriad of possible silica oligomers being formed. Infrared spectra also provide a distinction between linear and cyclic species, particularly as two different $\mathrm{Si}-\mathrm{O}-\mathrm{Si}$ asymmetric stretching modes ${ }^{16,18}$, but again this is only a coarse view of the material as it is hard to pin point the most likely species that are present. At a larger scale, it is often unclear to practitioners how the silica gel network is being formed in solution, and how that process is influenced by changes in reaction conditions or in chemical composition of the starting precursors. The use of molecular simulation tools can provide valuable mechanistic insights that are essential for the rational design of these materials, while also providing a route to explore new formulations in a cost-effective manner.

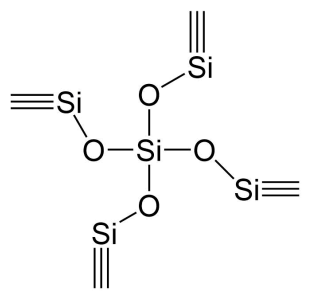

$\mathbf{Q}^{4}$<smiles>C#[Si]O[Si](O)(O[Si]=C)O[Si]=C</smiles>

$Q^{3}$

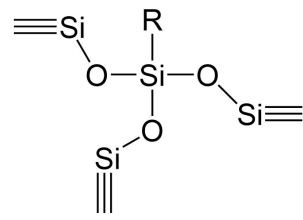

$\mathrm{T}^{3}$<smiles>C=[Si]O[Si](O)(O)O[Si]=C</smiles>

$\mathbf{Q}^{2}$<smiles>[R][Si](O)(O[Si]=C)O[Si]#C</smiles>

$\mathbf{T}^{2}$<smiles>C=[Si]O[Si](O)(O)O</smiles>

$\mathbf{Q}^{1}$<smiles>[R][Si](O)(O)O[Si]=C</smiles>

$\mathbf{T}^{1}$<smiles>O[Si](O)(O)O</smiles>

$\mathbf{Q}^{0}$<smiles>[R][Si](O)(O)O</smiles>

$\mathrm{T}^{0}$

Figure 3: The $\mathrm{Q}^{n}$ nomenclature used to identify Si environments, according to the number of siloxane bonds $(n)$ in which they are involved. Q designates a tetrafunctional Si atom, such as those arising from TMOS or TEOS precursors, and T represents trifunctional $\mathrm{Si}$ atoms, in which there is one non-reactive organic functionality, $R$ (e.g. a methyl group in the case of MTMS-derived structures). Adapted from Ref. 16. 
This work aims at providing an overview of the most recent molecular simulation studies (from the past 20 years) applied to silica aerogels, with emphasis on the techniques used and the main conclusions obtained. By the very multiscale nature of sol-gel chemistry different MM\&S methods need to be employed, each suitable for describing phenomena occurring at a different scale. This review is organized as follows. Apart from the current section which frames the topic, the next section (Section 2) will focus on studies involving quantum mechanics (QM) calculations, which are useful to model events at the atomic scale that include electronic effects. Section 3 will deal with classical molecular dynamics (MD) simulations, which can be used to study silica aerogels at the nanoscale, incorporating time as a fundamental variable. Finally, in Section 4, the extension of MD simulations to the mesoscale through the use of coarse-graining models is described, and the corresponding applications for modelling the silica aerogel 3D structure are discussed.

\section{Theoretical framework}

\subsection{Quantum mechanics methods}

Quantum mechanics (QM) is currently the most accurate physical model of matter at the fundamental level. In principle, the electron distribution of any molecular system can be obtained by solving the time-independent Schrödinger equation,

$$
\widehat{H} \psi(\mathbf{r})=E \psi(\mathbf{r})
$$

where $\psi(\mathbf{r})$ is the wave function (whose square gives the probability distribution of electrons in space), $\mathbf{r}$ is the vector containing the spatial coordinates of all particles forming the system (nuclei and electrons), $E$ is the energy of the system, and $\widehat{H}$ is the Hamiltonian operator which contains the description of the two main energy components of the system, kinetic and potential, the latter arising from the electrostatic interactions between all particles of the system. Because of the complexity of $\widehat{H}$ for many-body sys- 
tems, several approximations are required to solve Equation 1. The Born-Oppenheimer approximation, assumed in all major QM-based numerical methods, is based on the large difference between nuclei and electron masses, and considers the nuclei to be stationary and the motion of the electrons to depend only parametrically on the nuclear coordinates, which greatly simplifies $\widehat{H}$ and allows a Schrödinger equation for the electrons to be solved separately. ${ }^{19}$

Furthermore, an approximate description of the electronic structure of the system is required. In the context of molecular orbital theory, the electron wave functions of a molecule (molecular orbitals, MO) can be approximated as a linear combination of the atomic orbital basis functions (LCAO-MO approach) ${ }^{20}$, as follows:

$$
\psi=\sum_{i=1}^{N} a_{i} \phi_{i}
$$

in which $\psi$ is the molecular wave function, $a_{i}$ are coefficients determined iteratively by a proper algorithm, and $\phi_{i}$ are the atomic wave functions. The latter are expressed as a set of mathematical basis functions, or basis set. These basis functions are usually in the form of a combination of Gaussian functions - contracted Gaussian orbitals (CGTO) - as they provide the best compromise between accuracy and computational efficiency. ${ }^{20,21}$ The number of basis functions assigned to each orbital (2 - double zeta, 3 - triple zeta, etc.) has a profound impact on the accuracy of the results, but it is common to assign more basis functions to valence orbitals than to core orbitals to improve performance - this is the rationale behind the split-valence scheme. Double and triple zeta split-valence Pople basis sets are the most used for energy and spectroscopic calculations in this field ${ }^{22-29}$.

Alternatively, the plane wave approach can be used, in which complex exponentials, or plane waves, are the key functions of the basis set. The size of the set is then determined by considering all possible plane waves up to a pre-determined energy cut-off. ${ }^{20}$ Due to the nature of plane waves this approach is particularly suitable for periodic systems. However, such a basis set can become very expensive to compute if all electrons are being considered. Instead, the effect of core electrons, where density varies the most but which often contribute the least to chemical properties, can be modeled as an average effective 
potential, or pseudopotential, leaving valence electrons to be described by plane waves. ${ }^{30}$ A detailed description of the theoretical aspects and applicability of the various existing basis sets and pseudopotentials is out of the scope of this work and can be found in other reviews. ${ }^{20,31}$

Numerical solutions to the electronic Schrödinger equation can proceed by iteratively updating the orbital coefficients $a_{i}$ (Equation 2) until a energy tolerance is met, according to the Self Consistent Field (SCF) algorithm, as first devised in the method of Hartree-Fock (HF). Nevertheless, obtaining these solutions in an accurate manner is not straightforward, mainly because of the existence of electron correlation which is ignored in the HF formalism. ${ }^{32}$ Ab-initio extensions to the HF theory that include electron correlation (post-HF methods) are in most cases prohibitive to compute, as they present polynomial and even exponential scaling with the number of orbitals. Density functional theory (DFT) ${ }^{32,33}$ is currently the most popular approach to tackle this problem. Overall, it corresponds to a reformulation of QM that revolves around the concept of electron density, $\rho(\mathbf{r})$, instead of the traditional (but abstract) wave function. In principle, the electron density contains all the information required to build the Hamiltonian, while greatly reducing the number of variables. The result is a good compromise between chemical accuracy and computational effort. The energy of the system is a functional of the electron density, according to the Kohn-Sham (KS) equation,

$$
E[\rho(\mathbf{r})]=T_{s}[\rho(\mathbf{r})]+J[\rho(\mathbf{r})]+E_{x c}[\rho(\mathbf{r})]+\int \rho(\mathbf{r}) V(\mathbf{r}) d \mathbf{r}
$$

where $T_{s}$ and $J$ are, respectively, the functionals of kinetic energy and Coulomb repulsion for non-interacting electrons, $E_{x c}$ the exchange-correlation $(\mathrm{XC})$ functional containing all electron correlation corrections, and $V(\mathbf{r}$ the potential energy from the nuclei. The KS equation can be solved iteratively using a SCF approach similar to that of HF-based methods.

While the KS equation contains no approximations in its derivation, the exact form of the XC functional is not known. ${ }^{33,34}$ As such, approximations have to be made, and intense research has been devoted to the development of functionals of high accuracy for 
DFT. These generally fall into 3 categories $^{33}$ : i) Local Density Approximation (LDA) functionals, which assumes a uniform electron density in space (a very rough approximation); ii) Generalized Gradient Approximation (GGA) functionals, which includes corrections to LDA taking the gradient of electron density into account, or alternatively the kinetic energy (meta-GGA); and iii) hybrid functionals, that are built as a weighted sum of different functionals and also a HF energy term. From the plethora of DFT functionals available today, the most widely used by far is the hybrid Becke 3-parameter Lee-Yang-Parr (B3LYP) functional, due to its broad applicability with generally good accuracy. Nevertheless, the development of functionals of increased accuracy and wide applicability is a highly active topic to this day. ${ }^{34-37}$

The methodologies described so far are proficient for obtaining equilibrium structures of molecules, from which a wide range of chemical properties can be estimated with reasonable accuracy (depending on the level of theory): thermochemical data, IR and NMR spectra, band gap energies, transition states of reactions, etc. ${ }^{38,39}$ It is also possible to simulate the dynamics of a chemical system by performing ab initio molecular dynamics (AIMD) calculations, which are particularly useful for studying the mechanisms and kinetics of chemical reactions. The main difference between AIMD and classical MD (which will be described in more detail later) is the description of the potential energy of the system: while classical MD resorts to simple empirical potentials to describe atomic interactions, with only averaged electronic descriptions, AIMD computes such interactions explicitly from the detailed electronic structure of the atoms. As expected, this added complexity results in exponentially longer calculation times compared to classical MD. Typically, only a few hundreds of atoms and tens of picoseconds can be realistically simulated.

The time-dependent Schrödinger equation combined with the Born-Oppenheimer approximation for separation of nuclei and electron motion provides both a theoretical background and practical approach to perform AIMD simulations. The positions of the nuclei can be updated at each time step using Newton's Second Law, with the forces that act on each atom being derived from SCF energy calculations (from the time-independent 
Schrödinger equation) on-the-fly. This is the basic idea behind Born-Oppenheimer molecular dynamics (BOMD). ${ }^{30,40}$ However, to perform QM calculations at every time step can get prohibitively expensive. The seminal contribution of Car and Parrinello alleviated this issue by producing a more operable method to conduct AIMD simulations, which is known as Car-Parrinello molecular dynamics (CPMD). ${ }^{30}$ In CPMD the electron orbitals are introduced explicitly as degrees of freedom of the system dynamics, in the form of a fictitious kinetic energy term for the orbitals (with fictitious mass $\mu$ ) added to the system's Lagrangian. Both the nuclei positions and orbital coefficients are evolved in time by solving the respective equations of motion (from the Euler-Lagrange formalism). Now recalling the Born-Oppenheimer approximation, since the movement of the electrons is assumed to be essentially independent of the movement of the nuclei (occurring with different energy scales), it is supposed that the subsequent electronic configurations propagated this way will not deviate substantially from those of the true Born-Oppenheimer energy surface, with the error remaining bounded, and this obviates the need to keep doing SCF calculations at every time step. ${ }^{30}$

\subsection{All-atom molecular dynamics simulations}

While QM methods can provide unparalleled accuracy, they require extraordinary computational resources, and the scaling with system size prevents, at least currently, the study of anything beyond the scale of silica primary particles. To capture phenomena occurring at the spatial and temporal nanoscales, all-atom classical MD simulations are the most appropriate approach. Starting from a box of $N$ atoms in a known configuration, the positions, $r_{i}$, and velocities, $v_{i}$, of every atom $(i)$ of the system can be known by integration of Newton's Second Law with respect to time,

$$
F_{i}=m_{i} \frac{\mathrm{d} v_{i}}{\mathrm{~d} t}=m_{i} \frac{\mathrm{d}^{2} r_{i}}{\mathrm{~d} t^{2}}
$$


with the forces $F_{i}$ acting on atom $i$ being related to the potential energy of the system, $U$, by:

$$
F_{i}=-\frac{\partial U}{\partial r_{i}}
$$

Periodic boundary conditions (PBC) are typically applied to the simulation box, whose dimensions should then obey the minimum image convention to prevent self-interaction artifacts. ${ }^{19,41}$ The time integration is done numerically, for which several algorithms have been developed over the years ${ }^{19,41}$, the most commonly used in silica aerogel simulations being the leapfrog and velocity Verlet algorithms, employing time steps of 0.5 to 1 fs with no bond restrictions. ${ }^{42-53}$

$U$ contains the information about the physical and chemical interactions between the atoms of the system. The equations that describe all these interactions are referred as force fields $(\mathrm{FF})$, and correspond to simple empirical formulas whose computation is relatively inexpensive when compared to QM. The most challenging procedure during the preparation of a MD simulation is to find the suitable parameters for such equations that more accurately describe the physics/chemistry of the system under study. This can be done by fitting the equations to experimental data or by performing QM calculations, but there is no universal procedure to do such a fitting. In any case, the reliability of MD simulations critically depends on the quality of FF. Physical FFs possess the simplest mathematical descriptions, as they are meant to describe only physical bonded and nonbonded interactions between atoms. The general format of a physical FF is given by ${ }^{21}$ :

$$
\begin{aligned}
U=\left.U\left(r_{\mathrm{AB}}\right)\right|_{\text {stretch }}+U( & \left.\theta_{\mathrm{ABC}}\right)\left.\right|_{\text {angular }}+\left.U\left(\varphi_{\mathrm{ABCD}}\right)\right|_{\text {torsion }}+\left.U(r, \theta, \varphi)\right|_{\text {combined }}+ \\
& +\left.U\left(r_{\mathrm{AB}}\right)\right|_{\mathrm{vdW}}+\left.U\left(r_{\mathrm{AB}}\right){ }_{\mathrm{AB}}\right|_{\text {electrostatic }}+\left.U\left(r_{\mathrm{XH}}\right)\right|_{\mathrm{H}-\text { bond }}
\end{aligned}
$$

where $\left.U\left(r_{\mathrm{AB}}\right)\right|_{\text {stretch }},\left.U\left(\theta_{\mathrm{ABC}}\right)\right|_{\text {angular }}$ and $\left.U\left(\varphi_{\mathrm{ABCD}}\right)\right|_{\text {torsion }}$ are the bonded energy components associated with bond stretching, angular deformation and bond torsion, respectively, whereas the term $\left.U(r, \theta, \varphi)\right|_{\text {combined }}$ accounts for coupling between different modes of vibration. $\left.U\left(r_{\mathrm{AB}}\right)\right|_{\mathrm{vdW}},\left.U_{\mathrm{AB}}\right|_{\text {electrostatic }}$, and $\left.U\left(r_{\mathrm{XH}}\right)\right|_{\mathrm{H}-\text { bond }}$ are the non-bonded components, corresponding to the van der Waals, electrostatic and hydrogen bond interactions, respectively. A number of physical FFs for silica materials have been developed. For 
instance, the INTERFACE FF is a FF created by Heinz et al. ${ }^{54}$ to describe a number of inorganic surfaces, including amorphous silica surfaces. The FF was parameterized in a thermodynamically consistent way and validated extensively against experimental values of bulk and surface properties (getting closer to those values than other physical FFs). INTERFACE was also designed to be transferable, i.e. capable of being used in conjunction with organic systems, as it shares the same general format (Equation 6) with biomolecular FFs (e.g. CHARMM). The CLAYFF ${ }^{55}$ and GLASSFF ${ }^{56}$ FFs are other notable examples of silica physical FFs.

The main limitation of physical FFs is their inability to model chemical phenomena, since the bonds between atoms and their partial charges are fixed throughout the simulation. To circumvent this problem, more complex FFs were developed which can be classified as reactive FF. Reactive FFs are those which either do not consider explicitly a distinction between bonded and non-bonded atoms or allow for the dynamic breaking and formation of bonds, described by simple heuristics to emulate QM results. The main reactive $\mathrm{FF}$ that have been described in the literature for amorphous silica-based materials are:

i) The atomic potential of Feuston and Garofalini ${ }^{57,58}$, which can be considered a reactive FF since no distinctions are made between bonded and non-bonded interactions; instead, the total potential energy is the sum of the two-body Born-Mayer-Huggins $(\mathrm{BMH})$ potential (short range repulsion and Coulomb interaction, modified with an extra term to describe interchangeability of $\mathrm{O}$ atoms between water and silica), and a three-body potential to produce correct $\mathrm{Si}-\mathrm{O}-\mathrm{Si}$ and $\mathrm{O}-\mathrm{Si}-\mathrm{O}$ bond angles.

ii) The potential developed by Vashishta et al. ${ }^{59}$ is analogous to the Feuston-Garofalini potential since it is also made from two and three-body potentials, but possesses an additional charge-dipole interaction term on the two-body potential, in order to account for the polarizability of $\mathrm{O}$ atoms on siloxane bonds, which possess a strong ionic character.

iii) The van Beest, Kramer and van Santen potential (BKS $)^{60}$, a two-body reactive potential in which the total interaction between two atoms is defined as the sum of the 
Buckingham and Coulomb potentials. Its simplicity (with only five parameters) results in big performance gains compared to the previous potentials, while it is still able to reproduce the structure of silica materials with reasonable accuracy. Since it does not reproduce very well some thermodynamic properties, such as phase diagrams, Carré et al. ${ }^{61}$ improved the BKS FF in that respect by a reparametrization to fit CPMD simulation data (the authors called this FF CHIK). In another publication ${ }^{62}$, these authors also introduced a more computationally efficient version of the BKS potential, by truncating and smoothing the Coulomb interactions in such a way as to reproduce (and therefore replace) a more expensive Ewald summation. This modified version, which employs the Wolf method for the treatment of the electrostatic potential, is called Wolf-BKS. ${ }^{62}$

iv) A version of the Tersoff potential, originally developed for silicon, and re-parametrized for silica systems by Munetoh et al. ${ }^{63}$ This is a reactive potential of two-body terms, whose parameters were obtained for $\mathrm{Si}$ and $\mathrm{O}$ atoms and $\mathrm{Si}-\mathrm{O}$ bonds in such a way as to accurately reproduce DFT calculations and experimental crystallographic data.

v) ReaxFF, a reactive FF parameterized for silica systems (ReaxFFSiO) by van Duin et al. ${ }^{64}$ This FF, unlike the previous ones, explicitly accounts for bond breaking and formation by computing the bond order (BO), a dynamical parameter used to describe single, double and triple covalent bonds based on inter-atomic distances. The bonded part of the potential energy of ReaxFF is a function of this parameter and includes other components to correct the geometry of the formed molecules (lone pair, bond angle and bond torsion energies) and coordination of the atoms. Partial charges are also dynamical, and are updated by the electronegativity equalization method. ${ }^{65}$ The ReaxFFSiO FF was parameterized exclusively with DFT data. ${ }^{64}$ The original FF was later extended by Fogarty et al. ${ }^{66}$ to describe water-silica interactions. ReaxFF has enjoyed great success in describing sol-gel condensation reactions and silica surface properties at a fraction of the computational cost of equivalent AIMD simulations (but still much slower than simulations with other classical FF described here). ${ }^{67-70}$

The functional equations of the FFs mentioned above can be found review articles by Yeo et al. ${ }^{71}$ and Côté et al. ${ }^{72}$, and for more details on theoretical and technical aspects 
of MD simulations in general the reader is referred to other works. ${ }^{19,21,41,73,74}$

\subsection{Coarse grained molecular dynamics simulations}

In spite of the dramatic growth in computational power in the near past, all-atom MD simulations can still only realistically achieve hundreds of nanoseconds of physical simulation time and/or treat systems with up to $10^{5}-10^{6}$ atoms. This is insufficient for an adequate sampling of slower large scale degrees of freedom of the system which, in silica aerogels, are intimately linked with the porous pearl necklace structure, and significantly impact structural and mechanical properties. As pointed out by Ferreiro-Rangel and Gelb $^{75}$, aerogels of low density have characteristically long relaxation times, and an accurate representation of their mechanical behavior needs to take such effects into account. Furthermore, it is expected that the quality of the predictions of structural, mechanical and thermal properties of silica aerogels can improve when a larger system is considered.

To achieve the goal of simulating mesoscale systems is necessary to undertake simplifications in the underlying physical model. In the same manner as the transition from the quantum models to the classical models implies a loss in detail of the electronic structure (which is replaced by an averaged representation, by means of the FF), it is possible to go one step further and replace a set of atoms in a MD simulation by a single pseudoatom, called a bead, thus reducing the overall number of particles and greatly simplifying the interaction potentials and smoothing the energy landscape due to the exclusion of fast degrees of freedom. ${ }^{76,77}$ This is the philosophy underlying coarse-graining MD (CG-MD) simulations, which can thus provide access to time scales of $10^{-6}$ to $10^{-3}$ seconds thanks to much higher time steps, and tackle systems containing millions of particles. ${ }^{77,78}$ This approach has been successfully applied to study various molecular systems, such as biomolecules, organic liquids, polymers, electrolytes, etc. ${ }^{76-79}$ A more detailed description of these methods goes beyond the scope of this work, but it can be found in other publications. ${ }^{76-79}$

The first CG model of a silica aerogel targeting MD simulations was developed 
by Gelb ${ }^{80}$. Until then, numerical simulations were the only approach used to study silica aerogels at the mesoscale by considering silica primary particles as the fundamental structural units. ${ }^{81-88}$ The gel network in these simulations was constructed via the diffusion-limited cluster-cluster aggregation (DLCA) model, which simulated the Brownian motion of particles and assumed irreversible bonding between particles upon collision. Aerogels generated by DLCA have been found to reproduce well the fractal structure of silica aerogels and to make predictions of the small angle neutron scattering (SANS) spectra in good agreement with experiments. ${ }^{81-83,85}$ However, DLCA-based models alone are not adequate to obtain mechanical properties of silica aerogels because they rely on rigid bonds between particles which cannot describe the deformations taking place under strain. The CG-MD model proposed by Gelb ${ }^{80}$ was intended to circumvent this issue by considering spherical primary particles with the density of amorphous silica $\left(2.2 \mathrm{~g} \mathrm{~cm}^{-3}\right)$ as the CG beads, and by suggesting a simple FF to describe the interparticle bonded and nonbonded interactions, with the former being the sum of bond, angle and torsion terms, and the latter corresponding to the van der Waals interaction (i.e. the first three terms and the fifth term in Equation 6). In this model, bonds can be created and broken dynamically. Whenever two particles come within a certain distance $r_{b}$ of each other a random algorithm decides whether a bond is formed or not, by comparing a randomly generated number with a probability threshold assigned beforehand. Two points are created on the surface of the particles, along the axis connecting their centers, and the bond is established between these two points, as represented in Figure 4. The bond energy between two particles $i$ and $j$ is described by a Morse potential ${ }^{80}$ :

$$
U_{\text {bond }}\left(r_{i j}\right)=E_{0}\left[\left(1-\exp \left(-k\left(r_{i j}-r_{b}\right)\right)\right)^{2}-1\right]
$$

where $E_{0}$ is the potential well depth. The bond is considered to be broken whenever its energy gets close to zero within a given tolerance. Bond angles $\left(\theta_{i}, \theta_{j}\right)$ are defined between the bond and the line demarcating the radius of the particle, while torsion angles $(\phi)$ are defined through auxiliary points on the surface of each particle, as exemplified in 

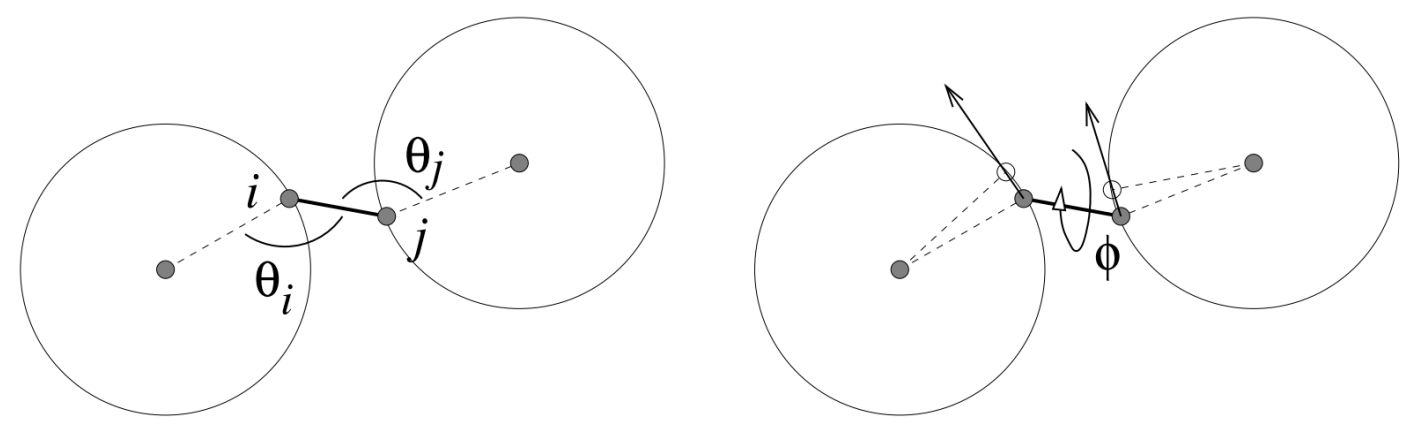

Figure 4: Schematic representation of the bonds, bond angles $(\theta)$ and torsion angles $(\phi)$ of the CG model proposed by Gelb. Reprinted (adapted) with permission from FerreiroRangel, C. A., Gelb, L. D., "Computational Study of Uniaxial Deformations in Silica Aerogel Using a Coarse-Grained Model", J. Phys. Chem. B, 2015, 119, 27, 8640-8650 ${ }^{89}$. Copyright (C)2015 American Chemical Society.

Figure 4. The potentials due to bond and torsion angles are defined as follows ${ }^{80}$ :

$$
\begin{gathered}
U_{\text {angle }}\left(\theta_{i}, \theta_{j}, r_{i j}\right)=K_{\theta}\left[\left|\cos \theta_{i}+1\right|^{X_{\theta}}+\left|\cos \theta_{j}+1\right|^{X_{\theta}}\right] \exp \left[A_{\theta} /\left(r_{i j}-r_{b}-r_{\max }\right)\right] \\
U_{\text {torsion }}\left(\phi, r_{i j}\right)=K_{\phi}\left|\cos \phi-\cos \phi_{0}\right|^{X_{\phi}} \exp \left[A_{\theta} /\left(r_{i j}-r_{b}-r_{\max }\right)\right]
\end{gathered}
$$

Finally, the van der Waals interaction term is a simple shifted Lennard-Jones potential ${ }^{80}$. A structure-based parameterization was done, albeit only semiquantitatively, by combining experimental information with data from QM and MD calculations, while also providing estimates to certain parameters in order to obtain physically reasonable forces.

It should be noted that the aforementioned issues of the DLCA procedure to model mechanical properties of silica aerogels have also been addressed within the context of numerical simulations. An extension of the DLCA method, named dangling bond deflection (DEF), was developed by Ma et al. ${ }^{86}$, in which branches in the 3D network, formed in excess during DLCA, are terminated to form a loop, by simulating thermal fluctuations around the bond connecting them to the main structure. Once the aerogel 3D network has been constructed by the DLCA-DEF method, finite element calculations can be performed to estimate mechanical properties, producing results that are well within the range of experimental values. ${ }^{86-88}$ For instance, the power law exponent values of the Young's modulus as a function of density predicted by Liu et al. ${ }^{87}$ using this model were in line with the experimental and theoretical values of silica aerogels of different densities 


\section{Computational studies of the sol-gel process}

\subsection{Atomic scale}

QM-based calculations have been extensively applied in the study of the initial steps of the sol-gel process, mainly concerning the mechanisms and energetics of the hydrolysis and condensation reactions. The four stages of the hydrolysis of TMOS were studied by Cheng et al. ${ }^{22}$, by DFT energy calculations at the B3LYP $/ 6-311+\mathrm{G}(\mathrm{d}, \mathrm{p})$ level of theory, with water treated by the implicit solvent model known as conductor-like polarizable continuum model (CPCM), in neutral, acid and basic media. It is now widely accepted that the mechanism of hydrolysis in acid medium consists of an initial $\mathrm{S}_{\mathrm{N}} 2$ attack of the nucleophile to the Si atom, forming a transition state where the Si atom is pentacoordinated, with simultaneous rupture of the $\mathrm{Si}-\mathrm{OCH}_{3}$ bond and subsequent formation of methanol. ${ }^{13}$ However, there were other alternative mechanisms proposed for acid hydrolysis, based on experimental data, such as that mediated by a siliconium ion, and other details such as the route of attack of the nucleophile - front-side, back-side of flank-side - remained contentious. ${ }^{13}$ As for the anionic mechanism, two alternatives have been proposed: a concerted $\mathrm{S}_{\mathrm{N}} 2$ mechanism analogous to that in acid medium, or a two-step mechanism with a stable intermediate bearing a pentacoordinated $\mathrm{Si}$ atom, with $\mathrm{S}_{\mathrm{N}} 2$ like kinetics. ${ }^{13}$. The neutral mechanism was still unknown. ${ }^{13,90}$ The computational study of Cheng et al. ${ }^{22}$ has unequivocally supported the concerted $\mathrm{S}_{\mathrm{N}} 2$ mechanism with the pentacoordinated-Si transition state for all three $\mathrm{pH}$ conditions, as specified as follows. 
i) In neutral medium, water acts as the nucleophile creating a $\mathrm{Si}-\mathrm{O}$ bond, with simultaneous proton transfer from the water molecule to the leaving methoxy group's O atom.

ii) In basic medium, a nucleophilic lateral attack is promoted by the $\mathrm{OH}^{-}$anion, forming the pentacoordinated Si structure. At the same time a proton is transferred from the $\mathrm{OH}$ group to the nearby methoxy group, which left after its respective $\mathrm{Si}-\mathrm{O}$ bond broke. However the known alternative of a back-side attack with inversion of the $\mathrm{Si}$ tetrahedron was not analyzed. ${ }^{13}$

iii) In acid conditions, a water molecule attacks one TMOS molecule, from the opposite side of an already protonated methoxy group, resulting in the ejection of an adjacent methoxy group. This corresponds to the widely accepted $\mathrm{S}_{\mathrm{N}} 2$ mechanism with the pentacoordinated-Si transition structure, although the authors of this study did not discuss the known inversion of the Si tetrahedron. ${ }^{13}$. An alternative flank side attack by $\mathrm{H}_{3} \mathrm{O}^{+}$on neutral TMOS was also considered by Cheng et al. ${ }^{22}$, but was found to have a higher energy barrier.

These mechanisms are represented in Figure 5. Cheng et al. ${ }^{22}$ also concluded that the hydrolysis of TEOS under neutral conditions proceeded by a similar mechanism to TMOS, but the calculated energy barrier was higher due to steric effects, a fact that is also known experimentally. ${ }^{13}$ These authors also found that activation barriers became slightly larger in neutral and acid medium after first hydrolysis but increased dramatically in basic medium. Elanany et al. ${ }^{90}$ also performed a study of the first hydrolysis of TMOS, using tight-binding AIMD simulations in vacuum. Both of the mentioned studies identified the catalytic effects of basic and acid conditions and fully agreed on the details of the neutral and basic mechanisms, with observation of transition structures with pentacoordinated $\mathrm{Si}$ atoms. ${ }^{22,90}$ However, for acid medium, Elanany et al. ${ }^{90}$ focused on the $\mathrm{S}_{\mathrm{N}} 2$ lateral attack of $\mathrm{H}_{3} \mathrm{O}^{+}$which had been ruled out by Cheng et al. ${ }^{22}$, and observed an ejection of the protonated methoxy group instead. The discrepancies between these works might be due to the different methods employed in each of them. Nevertheless, the results of Elanany et al. ${ }^{90}$ clearly discarded the mechanism proposal that involves a siliconium ion. 
(a)

DFT calculations at the B3LYP/6-31G(g) level of theory and with the polarizable continuum implicit solvent model (PCM) were used by Fernandez et al. ${ }^{23}$ in studying the mechanism of the last hydrolysis step of TEOS in basic conditions, in the presence of one and two water molecules. This investigation showed that the most favored mechanism was a $\mathrm{OH}$ attack from the opposite side of the leaving ethoxy group, generating a pentacoordinated intermediate, followed by proton transfer to the leaving group's $O$ atom, mediated by two water molecules in a hydrogen bond chain. Note that Cheng et al. ${ }^{22}$ had also considered the presence of one water molecule for the hydrolysis of TMOS in basic media and found it to be a less favorable route than that presented above, so it is interesting to realize that two water molecules can provide a favorable pathway for the hydrolysis of TEOS. The participation of extra water molecules in hydrolysis was also assessed for the last hydrolysis step of MTMS in neutral medium, as studied by Okumoto et al. ${ }^{24}$ by energy calculations at the B3LYP $/ 6+31 \mathrm{G}(\mathrm{d})$ level of theory, with and without the implicit PCM model for water. These authors found that the extra water molecules lowered the activation energy of the reaction by forming a hydrogen bond chain that facilitated proton transfer, with three water molecules in total forming the most stable intermediate complexes. Front-side nucleophilic attacks were taking place in 
this mechanism. It should be noted that these authors also found a $\mathrm{S}_{N} 2$ back-side attack mechanism for the last hydrolysis of MTMS in acid medium, just as Cheng et al. ${ }^{22}$ had found for TMOS (see above), but in basic medium they found a two-step mechanism involving water molecules and a stable intermediate with a pentacoordinated Si atom, in contrast to the concerted and non-mediated $\mathrm{S}_{\mathrm{N}} 2$ mechanism of Cheng et al. ${ }^{22}$.

The study by Cheng et al. ${ }^{25}$ on the basis of B3LYP $/ 6-311++\mathrm{G}(\mathrm{d}, \mathrm{p})$ calculations with the conductor-like screening implicit solvent model (COSMO) also helped in elucidating the mechanism for methanolysis of TMOS in neutral medium. In this case, the methanol's $\mathrm{O}$ atom attacked the $\mathrm{Si}$ atom forming a pentacoordinated transition state and the proton of the methanol hydroxyl group was transferred to the methoxy $\mathrm{O}$ atom (via hydrogen bonds), with simultaneous breaking of the $\mathrm{O}-\mathrm{CH}_{3}$ bond of that group. The leaving methyl group then left as dimethylether. However the calculated activation barrier of this reaction was extremely high $\left(283.6 \mathrm{~kJ} \mathrm{~mol}^{-1}\right.$, reducing slightly with methanolysis of further methoxy groups), and so this process was expected to only have a minor regulatory effect in normal sol-gel conditions. ${ }^{25}$

The mechanism of formation of dimers by condensation of hydrolyzed silica precursors (monomers) is also believed to involve a nucleophilic attack of one monomer's O atom on the Si atom of the other. ${ }^{13}$ However the details of the mechanism are different depending on whether the reaction medium is acid or basic ${ }^{13}$, with the mechanism in neutral conditions being unclear. The mechanisms under all of these conditions have been investigated by QM-based theoretical studies:

(a) In neutral medium, Cheng et al. ${ }^{22}$ identified a similar mechanism of condensation for the products of the four stages of TMOS hydrolysis $\left(\mathrm{Si}\left(\mathrm{OCH}_{3}\right)_{3} \mathrm{OH}, \mathrm{Si}\left(\mathrm{OCH}_{3}\right)_{2}(\mathrm{OH})_{2}\right.$, $\mathrm{Si}\left(\mathrm{OCH}_{3}\right)(\mathrm{OH})_{3}$ and $\left.\mathrm{Si}(\mathrm{OH})_{4}\right)$ : the $\mathrm{OH}$ group of one monomer establishes hydrogen bonds with a OR group $\left(\mathrm{R}=\mathrm{H}\right.$ or $\mathrm{CH}_{3}$ ) of the second monomer, which is followed by the formation of the $\mathrm{SiO}-\mathrm{Si}$ bond without a pentacoordinated intermediate, and migration of the $\mathrm{H}$ atom towards the OR group of the second monomer. These form a alcohol/water molecule that leaves after the $\mathrm{Si}-\mathrm{OHR}$ bond breaking (see illustration in Figure $6(\mathrm{a})$ ). Mechanisms involving rings of hydrogen bonds were found to be more 
favored. The activation barrier of condensation was larger than that of the neutral first hydrolysis, which suggested that multiple alkoxide groups can hydrolyze before any condensation reactions begin. The same mechanism has been reported by the study of Okumoto et al. ${ }^{24}$ for hydrolyzed MTMS dimerization, although these authors also have shown that two water molecules formed a hydrogen bonded ring with the

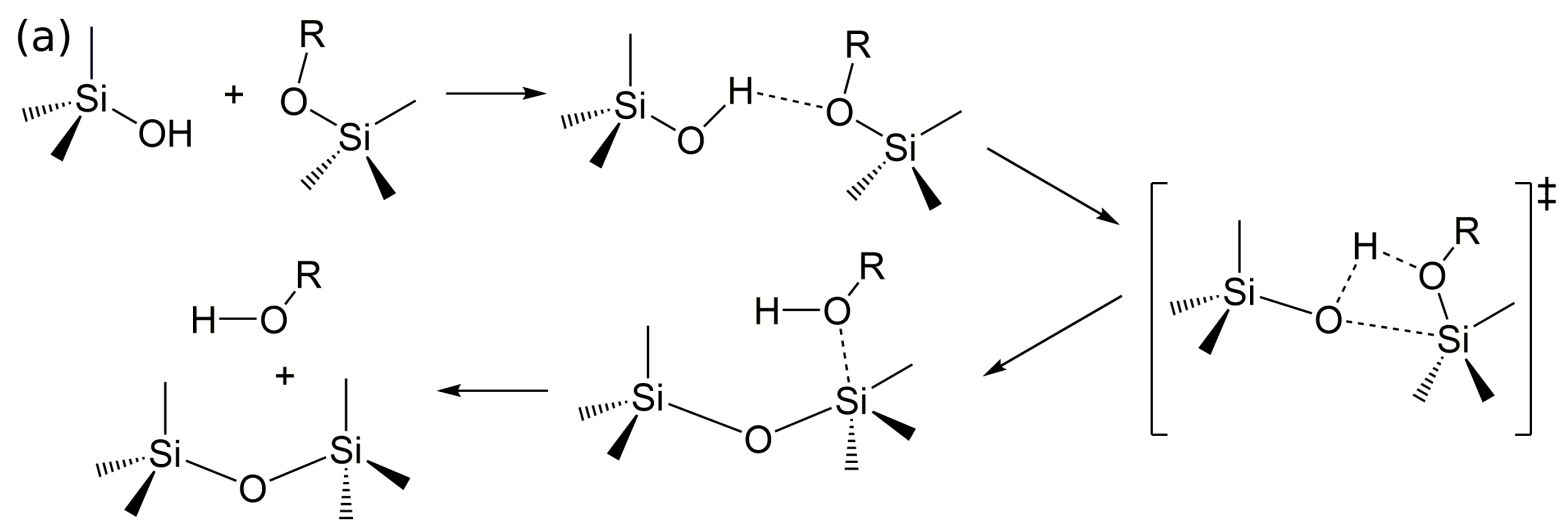

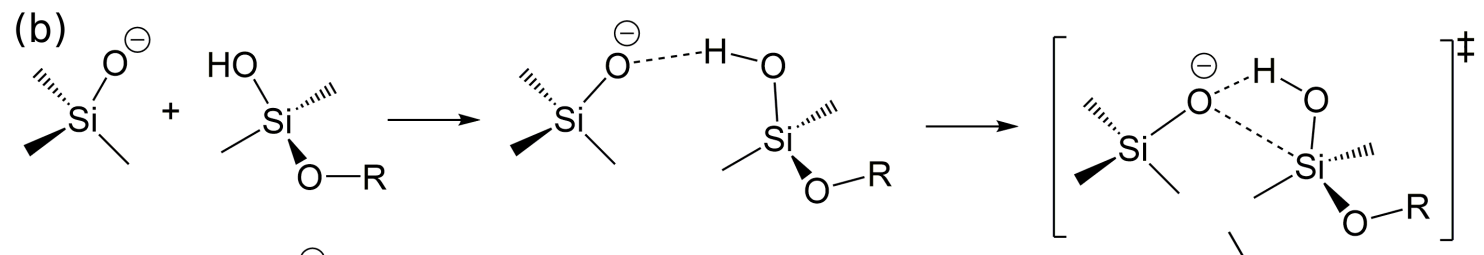<smiles>[R]O[Na]</smiles><smiles>CI</smiles>

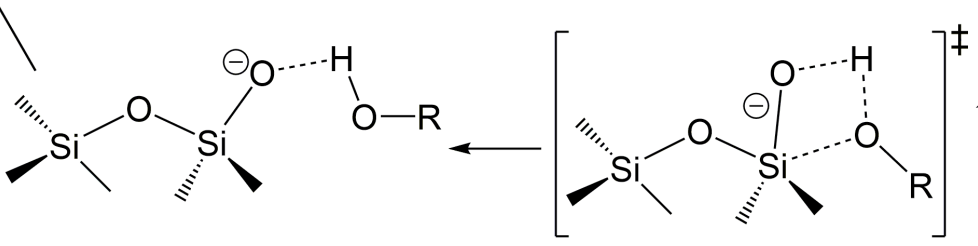

(c)<smiles>[R]O[Si][Si](C)(O)O[R]</smiles><smiles>[R]O[Si]1(C)O[C@@H]2O[SiH](C)(C)O[C@@H]21</smiles>

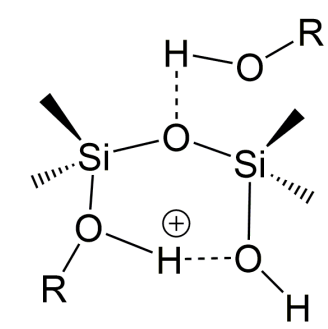

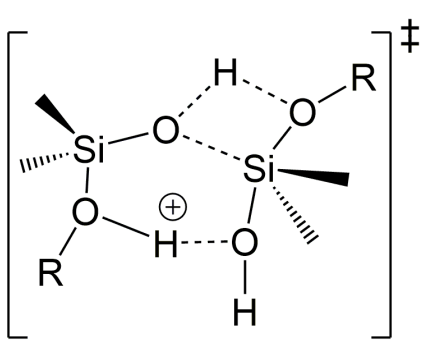

Figure 6: Schematic representation of the most favored mechanisms of TMOS condensation as identified in the work of Cheng et al. for neutral (a), basic (b) and acid (c) media. The symbol $\ddagger$ represents a transition state. Adapted from Ref. 22 . 
monomers which aided proton transfer. Trinh et al. ${ }^{91}$ also studied the condensation of $\mathrm{Si}(\mathrm{OH})_{4}$ via B3LYP $/ 6-31+\mathrm{G}(\mathrm{d}, \mathrm{p})$ calculations with the COSMO solvent model for water, and found a similar mechanism to Cheng et al. ${ }^{22}$, albeit with a larger energy barrier. All three studies mentioned here report the existence of a transition state bearing a pentacoordinated Si atom. ${ }^{22,24,91}$

(b) In basic medium, it is believed that the most likely mechanism of condensation is a back-side attack of an anionic species' $\mathrm{O}^{-}$atom (a monomer or oligomer) on the Si atom of a neutral monomer, forming the $\mathrm{SiO}-\mathrm{Si}$ bond. ${ }^{13}$ Whether the mechanism involved two steps with a stable intermediate species or one concerted step (both with $\mathrm{S}_{\mathrm{N}} 2$-type kinetics) is a question that remains unresolved. ${ }^{13}$ The two-step mechanism has been verified by multiple theoretical works for the condensation of $\mathrm{Si}(\mathrm{OH})_{4}{ }^{22,26,27,91}$, and has been extended to the possible products of incomplete hydrolysis of TMOS in the work of Cheng et $\mathrm{al}^{22}$. As presented in Figure 6 (a), the first step is the formation of the $\mathrm{SiO}-\mathrm{Si}$ bond, and the second step involves a proton transfer from a $\mathrm{OH}$ group (turning to an anion) to another vicinal $\mathrm{OH}$ group, which leaves as water. The energy barrier of dimerization was lower for monomers with a higher degree of hydrolysis, thus favoring branching in basic medium. It was concluded that the sequential mechanism, with the formation of an intermediate species possessing a pentacoordinated Si atom, was energetically more favorable than the concerted $\mathrm{S}_{\mathrm{N}} 2$ mechanism. All other theoretical works have proved the existence of this stable pentacoordinated intermediate. ${ }^{26,27,91}$

(c) In acid conditions, the generally accepted mechanism is a $\mathrm{S}_{\mathrm{N}} 2$ attack of a neutral monomer onto a protonated species. ${ }^{13}$ This $\mathrm{S}_{\mathrm{N}} 2$ mechanism, through a back-side attack, was reported by Pereira et al. ${ }^{92}$ to be the most favorable in methanol, when compared to the alternative lateral attack mechanism. This study consisted of DFT energy calculations with the BLYP functional, a DNP basis set and the COSMO implicit solvent model. The monomer protonation was concluded to be more favorable than the dimer protonation, suggesting that it was more likely for the neutral species to be attacking the protonated monomers, an observation that is also consistent with experimental findings. ${ }^{13,92}$ Cheng et al. ${ }^{22}$ showed an analogous mechanism for the condensation of the products of all stages of hydrolysis of TMOS. At first, both protonated and neutral 
monomers approach by establishing two hydrogen bonds. Then, the $\mathrm{O}$ atom of one $\mathrm{OH}$ group attacks the opposing $\mathrm{Si}$ atom, establishing the $\mathrm{SiO}-\mathrm{Si}$ bond, after which the $\mathrm{H}$ atom moves towards a nearby $\mathrm{OH}$ group, leaving as water (from the opposite side of the initial protonated group), with the dimer remaining protonated. This mechanism is schematized in Figure 6 (c). The main difference between the works of Cheng et al. ${ }^{22}$ and Pereira et al. ${ }^{92}$ is the nature of the pentacoordinated Si species: the former predicts that this species is a transition state while the latter predicts that it is a stable intermediate. The differences could be attributed to the different models and solvent used, which in the case of Cheng et al. ${ }^{22}$ was B3LYP/6-31G(d,p) with the CPCM implicit model for water.

From what has been exposed so far, it becomes apparent that the inclusion of water molecules can lead to different mechanisms. Furthermore, solvation effects seem to substantially affect the energetics of the reactions. For instance, Henschel et al. ${ }^{26}$ studied the dimerization of orthosilicic acid $\left(\mathrm{Si}(\mathrm{OH})_{4}\right)$ in basic medium with B3LYP/6-31G(d) calculations, both in vacuum and with a implicit water model (COSMO). While the geometries of the species involved in the reaction - reactant complex transition state and product complex - were similar in both cases, the energies were considerably different, with the vacuum conditions resulting in a more exothermic reaction while the COSMO procedure resulted in structures whose energy was less dependent on the strength of hydrogen bonding. Trinh et al. ${ }^{93}$ took an explicit solvent approach to study the same problem by conducting steered CPMD simulations including 2-3 $\mathrm{Si}(\mathrm{OH})_{4}$ molecules, one of which ionized, and 64 water molecules, for 15 ps. They found a similar mechanism as they had found in their previous study ${ }^{91}$ using a implicit solvent model, but observed much lower energy barriers (ca. $-20 \mathrm{~kJ} \mathrm{~mol}^{-1}$ ), especially for the barrier of the second step (water removal). They also found the reaction to be endothermic, opposed to the implicit solvent models and contrary to experiment. This was also the case for the formation of the linear and cyclic trimers.

In the face of these disparities, McIntosh ${ }^{27}$ proposed a hybrid solvation approach, in which a number of water molecules was explicitly used alongside the dielectric back- 
ground provided by the implicit CPCM solvent model. The validity of this approach, in comparison with pure implicit or explicit solvation models, was extensively evaluated by studying the kinetics of dimerization of $\mathrm{Si}(\mathrm{OH})_{4}$ in basic medium with energy calculations employing the HF, DFT (B3LYP functional) and second order Møller-Plesset perturbation theory (MP2) methods, and the $6-31+\mathrm{G}(\mathrm{d})$ basis set. The values of activation energies and pre-exponential factors were found to vary slightly with the number of explicit waters. Activation energies were sensitive to the level of theory, but the authors were able to develop a methodology in which the energies at the higher levels of theory with hybrid solvation effects (more computationally costly) could be approximated by faster low-level calculations (HF) with no solvent effects, to which corrections for explicit and implicit solvation (also computed at a lower level of theory) could be applied. By examining the convergence in bond lengths, transition state imaginary frequencies, and partial charges for a variable number of explicit waters, it was established that eight $\mathrm{H}_{2} \mathrm{O}$ molecules corresponded to the best compromise between quality and computational effort. With this hybrid model it was found that the activation energy of the water removal step of the condensation reaction was significantly lowered compared to the other two models alone. This was explained by an additional stabilization of the leaving water provided by explicit waters, together with the better agreement with experimental information, thus suggesting that this activation barrier should be closer to that of the reverse reaction of siloxane bond hydrolysis. Nevertheless, the implicit solvation model alone was better than the hybrid model at describing the initial formation of the hydrogen bonded reactant complex that preceded the $\mathrm{Si}-\mathrm{O}$ bond formation. Using this fact, the heat of the condensation reaction was then much closer to the experimental value than any other solvation model. Further refinements to the kinetic model were made by the inclusion of corrections to account for anharmonicity, variational and quantum tunneling effects, and were used to simulate monomer concentration as a function of time for various $\mathrm{pH}$ values. The overall profile was qualitatively correct, although systematic deviations were found due to the limited scope of the model. The maximum rate of monomer consumption was found at $\mathrm{pH}=9.5$, also close to experimental values. ${ }^{27}$

Another sol-gel reaction whose mechanism was elucidated by QM calculations is the 
hydrolysis of the siloxane bond in the silica dimer (disiloxane). Cypryk and Apeloig ${ }^{28}$ studied this reaction in neutral and basic media by performing energy calculations at the B3LYP/6-311+G(2d,p), HF/6-31G(d) and MP4/6-31G(d) levels of theory, as well as varying the number of water molecules. The participation of several water molecules changed the neutral mechanism significantly: with up to two water molecules a front-side attack of a water molecule to the Si atom occurred (i.e. on the same side of the leaving group), while for four water molecules the mechanism involved a back-side attack with proton transfer to the leaving group mediated by a chain of water molecules linked by hydrogen bonds. The latter mechanism had a lower activation energy than in the cases of one and two water molecules, although only slightly lower than in the two-water case. In acidic medium, the QM calculations allowed to rule out a possible front-side attack in favor of a back-side attack of water, which in this case was facilitated energetically due to the protonation of the dimer (the activation barrier was $16-18 \mathrm{kcal} \mathrm{mol}^{-1}$ lower than in neutral medium). With four water molecules the activation barrier was lowered significantly, with the same chain of hydrogen bonded waters forming and aiding proton transfer. The proposed mechanisms for neutral and acidic medium are represented in Figure 7. When a silanol group was bonded to a Si atom of the dimer significant lowering of the energy barriers of hydrolysis was also verified for the cases of three and four water molecules, which was attributed to the participation of the $\mathrm{OH}$ group in the hydrogen bonded complex that was promoting the proton transfer.

QM methods have also been applied in the study of larger silica oligomers. One of the earliest examples is the two-part study of Pereira et al. ${ }^{94,95}$, in which DFT calculations (BHL local functional and BLYP and B3LYP functionals, with double and triple numerical basis sets) were used for an energetic study of a wide array of possible linear, cyclic, polycyclic and branched oligomers, including from two to eight Si atoms. While the accuracy of the models used was admittedly limited, this study could nevertheless give an account for the most stable oligomers that might be formed at the beginning of the sol-gel process. It was found that linear trimers, tetramers and pentamers adopt cycliclike conformations stabilized by hydrogen bonds, which could facilitate intramolecular condensation. The stability of the clusters, represented by the energy of condensation 


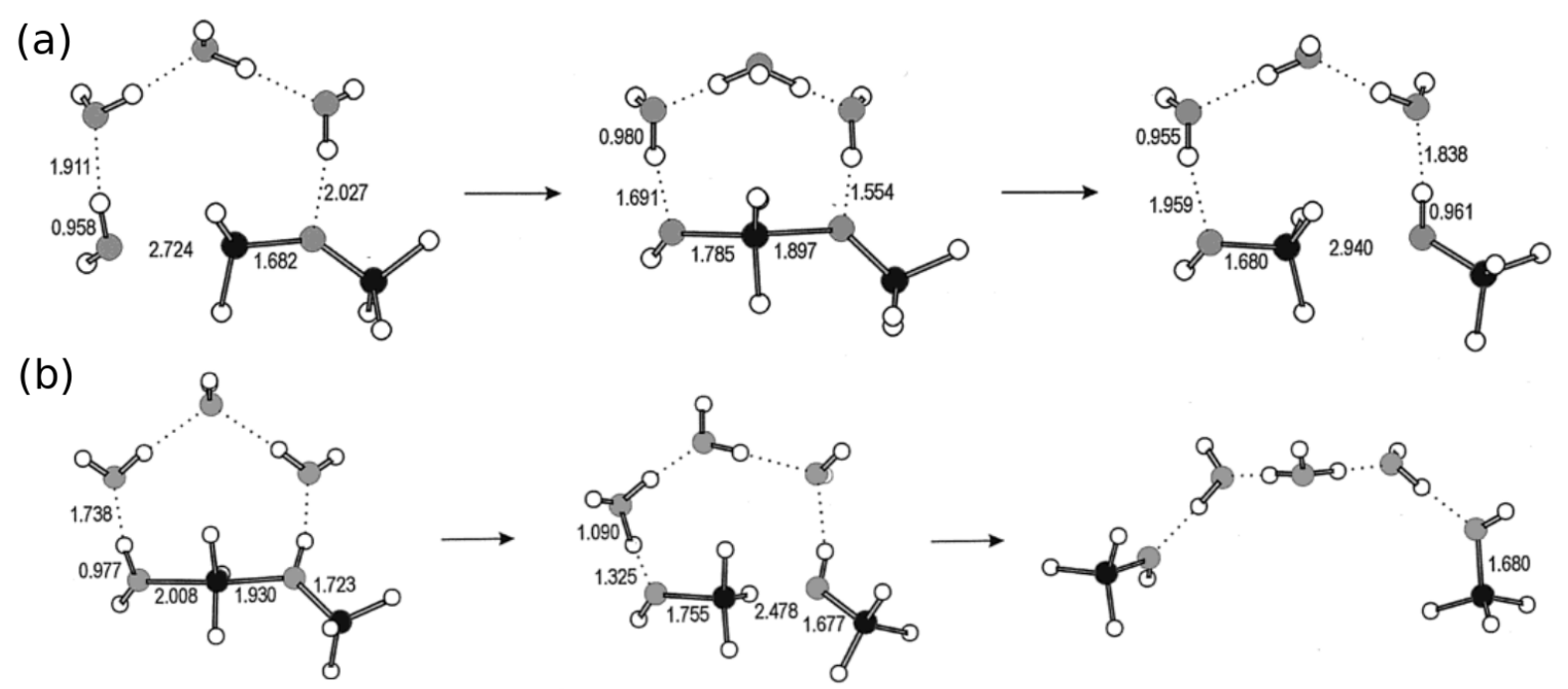

Figure 7: The most probable mechanisms of siloxane hydrolysis in neutral (a) and acidic (b) media, as identified by Cypryk and Apeloig. Reprinted (adapted) with permission from Cypryk, M. and Apeloig, Y., "Mechanism of the Acid-Catalyzed Si-O Bond Cleavage in Siloxanes and Siloxanols. A Theoretical Study",Organometallics, 2002, 21, 11, 2165 $2175^{28}$. Copyright (C)2002 American Chemical Society.

(normalized by the number of Si atoms of the oligomer), tended to increase with the degree of condensation for linear chains and to decrease slightly in the case of branched linear structures (vs. strictly linear ones). For cyclic clusters, condensation energy became more positive when ring strain was larger, but the stability was improved by the addition of side branches and the formation of stabilizing hydrogen bonds with the ring $\mathrm{OH}$ groups, as well as when cyclic hydrogen bond systems were present (such as, for instance, in the cyclic tetramer and hexamer). The energy of cyclic clusters was slightly larger than that of similar linear clusters, but tended to decrease with the size of the ring, converging towards the values of linear chains.

Most recently, McIntosh ${ }^{29}$ studied the mechanisms of formation of all possible clusters with up to four Si atoms in basic conditions by using the hybrid solvation approach described previously, and DFT energy calculations with the MP2/6-31+G(d)//HF/6$31+\mathrm{G}(\mathrm{d})$ model chemistry. He found that the thermodynamically most favorable route for linear trimer formation was the addition of a neutral monomer to a anionic dimer (instead of the reverse), with a similar two-step process ( $\mathrm{Si}-\mathrm{O}$ bond formation followed by proton transfer to the leaving $\mathrm{OH}$ group), via a pentacoordinated intermediate. Trinh et al. ${ }^{91}$ arrived to the same conclusion by an implicit solvent approach, and both studies 
results are consistent to what has been proposed based on experimental evidence ${ }^{13}$. In both works ${ }^{29,91}$, cyclization was then described to occur by intramolecular condensation (via the same mechanism), although the energy barrier for this process was higher than linear trimer formation due to conformational changes. However, two competing routes of similar energy barriers were found for the formation of the linear tetramer: i) addition of neutral monomer to anionic trimer, which could undergo cyclization to form the cyclic tetramer (this route is schematized in Figure 8); ii) addition of anionic monomer to neutral trimer, leading to a linear tetramer with the anionic $\mathrm{O}^{-}$atom in the middle of the chain, and therefore to the formation of the branched cyclic trimer upon cyclization. Another likely mechanism of formation of the branched cyclic trimer that was proposed consisted in the addition of anionic monomer to the cyclic trimer, which was a one-step process with low energy barrier, more favorable than the addition of neutral monomer to anionic cyclic trimer. Addition of anionic monomer to linear trimer was also referred as the most probable route for the formation of a branched trimer. The results of Trinh et al. ${ }^{91}$ also agree with this assessment, and although similar energy barriers were found in that study, the overall thermodynamics was different, showing again the effect of solvent models on energy calculations. The formation of bicylic tetramers was found by McIntosh $^{29}$ to be thermodinamically unfavorable, and the first activation barrier of the two-step process ( $\mathrm{Si}-\mathrm{O}$ bond formation) was very high due to an increase of ring strain. In this work, the use of the hybrid solvation model gave very different energies from the explicit and implicit models alone, but it actually allowed to predict the process of formation of the cyclic tetramer to be more energetically favored, which was better in line with the known prevalence of this structure experimentally. Thus, both of the works of McIntosh ${ }^{27,29}$ suggested that the use of a combination of implicit and explicit solvent models was a sound approach for studying reaction mechanisms due to its flexibility, as the explicit solvent molecules could best described short range effects within the first solvation shell, such as hydrogen bonding, while the implicit solvent model was best suited to describe long range solvent effects.

By using DFT calculations with implicit solvent (COSMO) or CPMD simulations with explicit water molecules, Zhang et al. ${ }^{96}$ were able to calculate reaction rate constants 
for the formation of several oligomers from a starting solution of $\mathrm{Si}(\mathrm{OH})_{4}$, which were used to build a kinetic model for a Monte Carlo simulation. It was reported that at

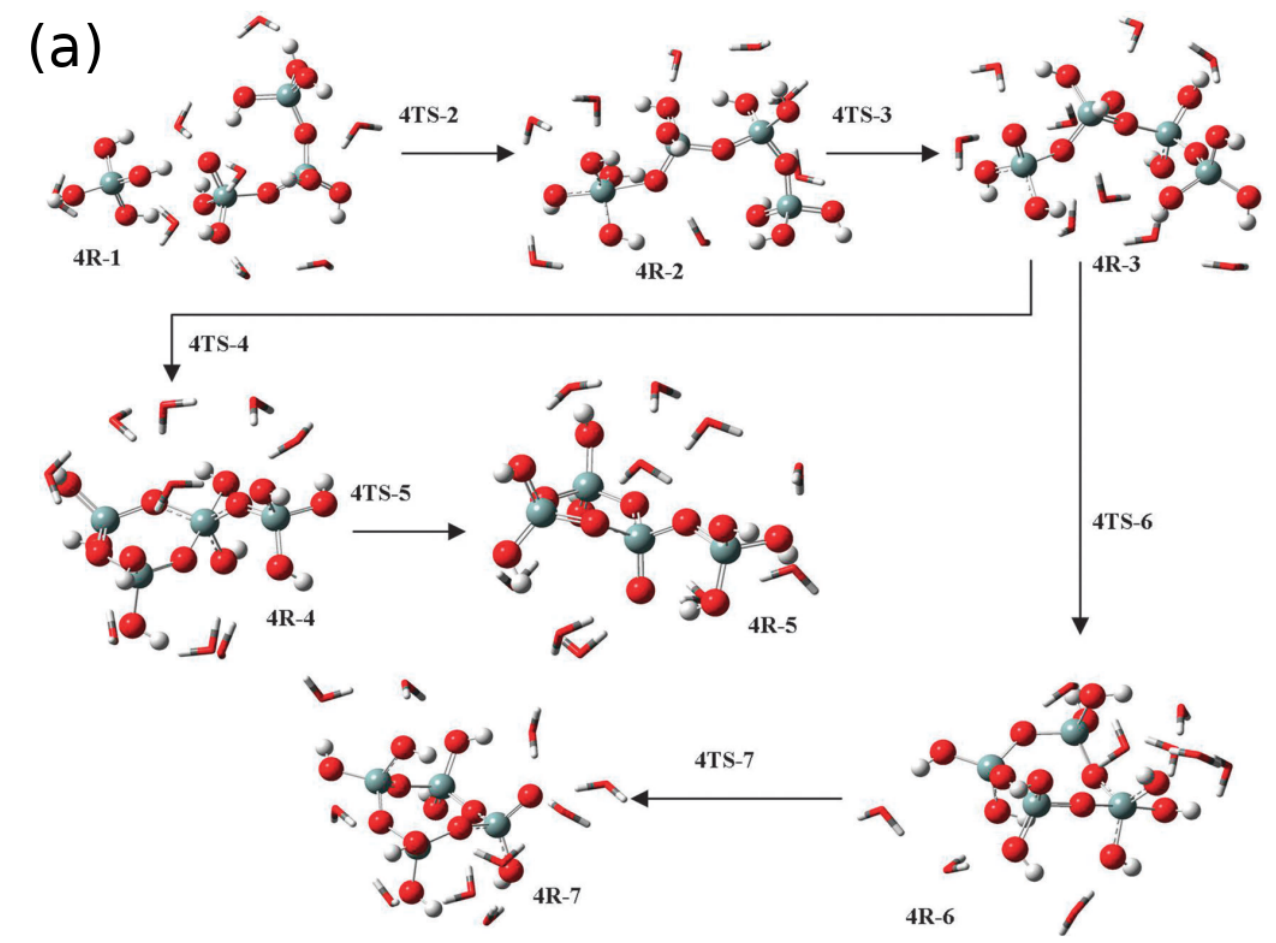

(b)

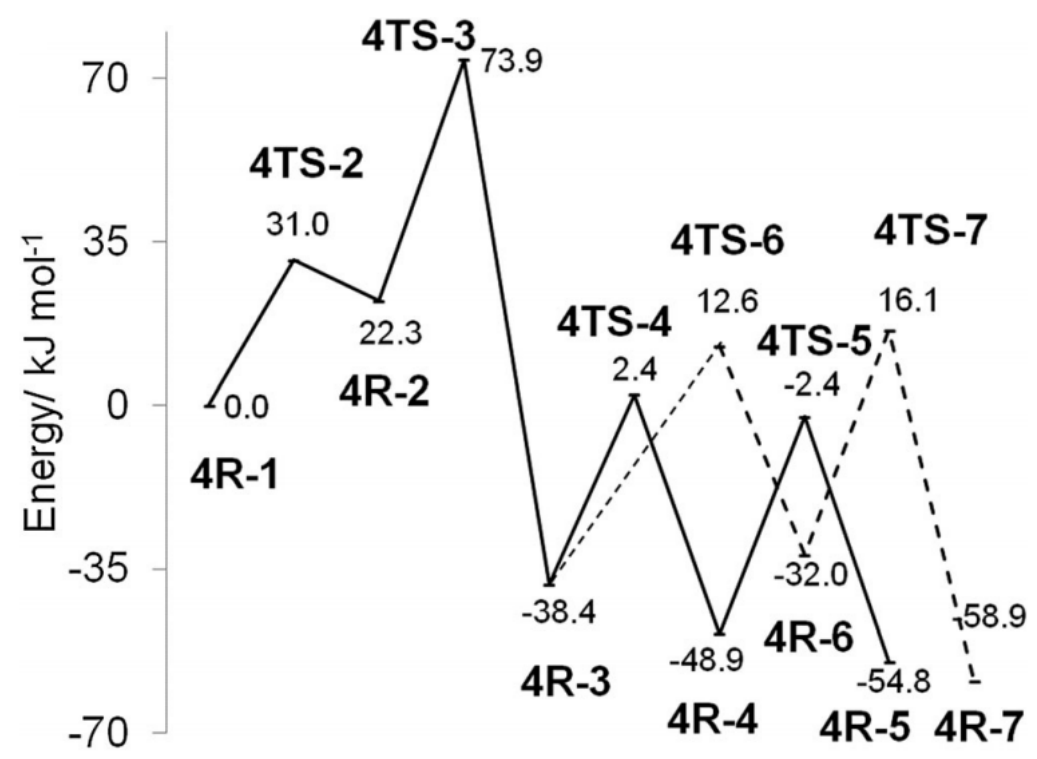

Figure 8: (a) Most likely routes predicted by McIntosh for the formation of linear tetramers and subsequent cyclization to form the cyclic tetramer or the branched cyclic trimer; (b) the respective energy surfaces along the reaction path (dashed line - cyclic tetramer; solid line - branched trimer). Republished with permission of the Royal Society of Chemistry, from Phys. Chem. Chem. Phys., "Theoretical investigations into the nucleation of silica growth in basic solution part I - ab initio studies of the formation of trimers and tetramers", McIntosh, G., vol. 15, 2013 ${ }^{29}$; permission conveyed through Copyright Clearance Center, Inc. 
$\mathrm{pH}=7$, in the initial stage of the polymerization process $(t<0.5 \mathrm{~s})$, the monomer was quickly consumed to form dimers, which were the dominant species early on. Dimers then rapidly led to linear and cyclic trimers in equal amounts and they were converted to linear tetramers after their concentration reached a peak early on $(0.5-1 \mathrm{~s})$. Upon cyclization, these finally gave rise to the cyclic tetramers which became the dominant species for the remainder of the reaction $(1-300 \mathrm{~s})$, reaching an equilibrium in which the concentration of most other species vanished. Similar profiles were obtained at higher $\mathrm{pH}$ values, but the characteristic time of each stage of the process was different. The initial stage of formation of linear species became slower, while the latter stage where cyclic tetramers were produced, became faster. The opposite occurred for acidic conditions. The maximum rate of polymerization (monomer consumption) was verified for $\mathrm{pH}=8$, close to experimental values and relatively close to the value predicted by McIntosh. ${ }^{27}$

Because QM calculations allow the prediction of spectroscopic quantities and their relationship with structural features, it is possible to make direct comparisons with experimental spectra which can better elucidate what kind of oligomer structures are being produced in the sol-gel process. For instance, by focusing only on the trimer and tetramer ring structures, for which IR and Raman spectra were calculated at the B3LYP/6-31G(d) level of theory, Monsivais-Gámez et al. ${ }^{97}$ were able to reproduce and interpret the main bands of the IR spectrum of a TEOS aerogel sample in the range $500-1500 \mathrm{~cm}^{-1}$, with good match between the theoretical and experimental frequencies. The Raman spectrum revealed two distinct peaks at 490 and $600 \mathrm{~cm}^{-1}$, which, according to the theoretical predictions, could be safely attributed to breathing vibration modes of the three and four-membered rings.

Ospino et al. ${ }^{98}$ used four different double cage silica oligomers derived from mixtures of the precursors TEOS and methyltriethoxysilane (MTEOS), optimized at the PBEPBE/6-31G(d,p) level of theory, to estimate IR frequencies, ${ }^{29} \mathrm{Si}-\mathrm{NMR}$ chemical shifts and inelastic scattering spectra (INS) frequencies. The good agreement between calculated and experimental values helped to interpret the experimental spectra, as it made possible to follow the changes in the spectral bands as the composition of the material 
changed. Furthermore, the authors observed a link between the changes in $\mathrm{Si}-\mathrm{O}-\mathrm{Si}$ bond lengths with addition of vicinal methyl groups and frequency shifts in the corresponding bands of the IR and INS spectra. B3LYP/6-31G(d) calculated frequencies also supported the analysis of experimental Raman spectra in the study of the hydrolysis of methylmethoxysilanes, of the form $\operatorname{Si}\left(\mathrm{CH}_{3}\right)_{x}(\mathrm{OH})_{4-x}(x=0$ to 3$)$, carried out by Bennett et al. ${ }^{99}$. By taking Raman spectra measurements of samples at different times along the hydrolysis process, it was possible to observe the formation of the successive hydrolysis products by the change in intensity of the respective peaks. The authors also explained that the decrease in frequency of the bands corresponding to $\mathrm{Si}-\mathrm{O}$ vibrations with the increase of methyl groups content was due to the lengthening of $\mathrm{Si}-\mathrm{O}$ bonds, as verified in the calculated equilibrium structures. They also ascribed the blue shift of these bands with increased degree of hydrolysis to the longer bond length and reduced masses of $\mathrm{Si}-\mathrm{OH}$ groups as opposed to $\mathrm{Si}-\mathrm{OCH}_{3}$ groups. ${ }^{99}$

Depla et al. ${ }^{17}$ also applied a combined theoretical and experimental Raman and ${ }^{29} \mathrm{Si}$ NMR spectroscopic analysis to study the distribution of reaction products of hydrolyzed TEOS $\left(\mathrm{Si}(\mathrm{OH})_{4}\right)$ condensation in acid medium, and the effects of different water:monomer ratios (W:M). It was found that for a $\mathrm{W}: \mathrm{M}$ value of 0.2 the rate of hydrolysis was insufficient for subsequent production of oligomers with complexity beyond linear trimers and tetramers. For $\mathrm{W}: \mathrm{M}=0.7$ there was a high concentration of linear chains, up to tetramers, followed by the cyclic species, in the order $4>5>6>3$-membered rings, and small amounts of branched oligomers. An increase in the value of W:M to 1.2 did not alter this distribution significantly, but led to a larger concentration of branched species. A kinetic model was derived, which predicted that, after an early step of rapid monomer consumption followed by dimer formation and subsequent consumption, the concentration of linear (first trimer then tetramer and pentamer) and cyclic products (in the order discussed above) increased steadily with time until reaching an equilibrium value. These profiles, shown in Figure 9, seem to be in agreement with those found by the kinetic model of Zhang et al. ${ }^{96}$. The concentration of branched species, while having a much smaller growth rate, grows almost linearly throughout the simulated time. Based on the observations of concentration profiles, the authors pointed out that the formation 

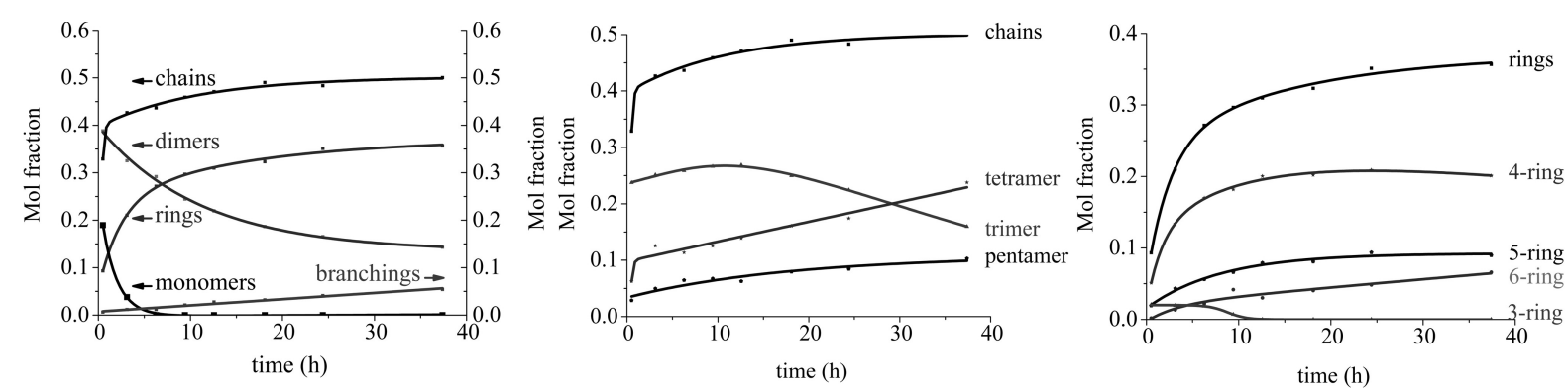

Figure 9: Time evolution of the concentration of the main products of orthosilicic acid condensation formed at the initial stage of the reaction, as predicted by the kinetic model developed by Depla et al. Reprinted (adapted) with permission from Depla, A., Lesthaeghe, D., van Erp, T. S., Aerts, A., Houthoofd, K., Fan, F., Li, C., van Speybroeck, V., Waroquier, M., Kirschhock, C. E. A., Martens, J. A., " ${ }^{129}$ Si NMR and UV-Raman Investigation of Initial Oligomerization Reaction Pathways in Acid-Catalyzed Silica SolGel Chemistry", J. Phys. Chem. C, 2011, 115, 9, 3562-3571 17. Copyright (C)2011 American Chemical Society.

of linear and cyclic oligomers was likely to be due to dimer plus dimer and dimer plus trimer additions, although no mechanistic evidence was provided. McIntosh ${ }^{29}$ showed $^{2}$ that dimer plus dimer addition was a possible route to cyclic tetramers, but his study was conducted for a basic medium.

Two comprehensive studies performed by Borba et al., first for MTMS-based aerogels ${ }^{100}$, and then extended to aerogels derived from the precursors TMOS and VTMS ${ }^{100}$, were aimed at determining the most probable silica oligomers that can be found in these materials. The undertaken approach was an energetic analysis complemented by a combined theoretical (B3LYP/6-311+ G(d,p)) and experimental analysis of IR and ${ }^{29}$ Si-NMR spectra (chemical shifts predicted at the B3LYP/IGLO-III level of theory). The energetic analysis provided the most stable configurations of a vast set of oligomers studied (from one to eight Si atoms), which corresponded to highly symmetric conformations in the cases of MTMS and VTMS, and low symmetry conformations in relation to TMOS. Large linear clusters assumed polycyclic-like conformations due to hydrogen bonding, with identical spectroscopic characteristics to cage structures. By calculating the condensation energy of each cluster (normalized by the number of Si atoms) and by comparing their theoretical IR spectra with the experimental counterparts, the authors concluded that MTMS aerogels were best described by a combination of cyclic oligomers and cage octamers, linear and cyclic oligomers for TMOS-based aerogels (in line with kinetic stud- 
ies previously mentioned above, for the basic conditions also used by Borba et al.) and a combination of linear, cyclic and cage structures for the VTMS-based material. The analysis of the ${ }^{29} \mathrm{Si}-\mathrm{NMR}$ spectra evidenced a predominance of highly ramified linear and cyclic structures due to the prevalence of the $\mathrm{Q}^{4}$ and $\mathrm{Q}^{3}$ peaks in the TMOS aerogel, while for MTMS and VTMS the presence of $\mathrm{T}^{3}, \mathrm{~T}^{2}$ and $\mathrm{T}^{1}$ peaks, and the comparison of NMR chemical shifts with calculated values, narrowed down the set of most probable species to the cage octamer and branched trimer for MTMS, and both of these and the branched tetramer for VTMS ${ }^{16}$

Maximiano et al. ${ }^{101}$ followed a similar approach to study organically-modified silica (ORMOSIL) aerogels derived from three different mixtures of precursors: $50 \%$ TMOS $/ 50 \%$ VTMS, 50\% tetraethylorthosilicate (TEOS)/50\% aminopropyltriethoxysilane (APTMS) and 50\% TEOS/50\% (3-glycidyloxypropyl)trimethoxysilane (GLYMO). An energetic analysis based on DFT B3LYP $/ 6-311+\mathrm{G}(\mathrm{d}, \mathrm{p})$ calculations yielded the most stable conformers of several possible structures for these mixtures, also pointing to species with high degree of condensation, particularly the cage octamers, as the most stable. It is noted that in the work of Maximiano et al. ${ }^{101}$ the combined experimental/computational analysis of IR spectra was done numerically, by fitting a weighted sum of the theoretical spectra of each silica species to the experimental data using a least squares optimization algorithm. As a result, theoretical spectra for the aerogel materials could be obtained, thus allowing to reproduce their experimental counterparts in a fairly good way. Furthermore, the least squares fitting procedure sieved the original set of energetically most stable silica species by identifying those that were the most representative of the aerogel. The same numerical analysis employed for aerogels of pure TMOS or VTMS yielded results in good agreement with those of Borba et al. ${ }^{16}$, who had performed a more qualitative comparison of theoretical and experimental IR spectra. In addition, analysis of experimental ${ }^{29} \mathrm{Si}-\mathrm{NMR}$ spectra, supported by chemical shifts calculated at the B3LYP/IGLO-III level of theory, generally corroborated the conclusions of the IR analysis. 


\subsection{Nanoscale}

\subsubsection{Structural properties and formation of silica aerogels}

The porous network structure of silica aerogels has been extensively studied by MD simulations. It is known from small-angle X-ray scattering (SAXS) and SANS measurements that silica aerogels present a fractal structure that is indicative of long range order within the length scale of secondary particles $(\sim 50 \mathrm{~nm}) .{ }^{9,102,103}$ The fractal structure arises from the aggregation of silica primary particles during the sol-gel process, and can manifest itself as mass and surface area correlation. As a result of the former, inside the fractal region the bulk density around a random point is known to have the following power law relationship with the distance from the point $(R)^{102}$ :

$$
\rho(R) \sim R^{d_{f}-3}
$$

where $d_{\mathrm{f}}$ is known as fractal dimension. On the other hand, from SAXS/SANS experiments, a curve of intensity $(I)$ vs. wavenumber $(q)$ in logarithmic coordinates includes three distinct regions, as shown in Figure 10-(a). ${ }^{9}$ The middle linear region of the graph $(1 / \xi<q<1 / a)$ corresponds to the fractal regime, in which the intensity decreases linearly with distance, according to a power law

$$
I(q)=q^{-d_{\mathrm{f}}}
$$

which means that $d_{\mathrm{f}}$ can be extracted directly from this region as the slope of the graph:

$$
d_{\mathrm{f}}=-\frac{\mathrm{d} \log (I(q))}{\mathrm{d} \log (q)}
$$

$\xi$ and $a$ demarcate the interval of distances for which fractal-like structural correlation exists: $a$ is the diameter of the primary particles and $\xi$ is the correlation length, the value beyond which the material is considered homogeneous (it is found to be equal to the typical size of secondary particles). ${ }^{9}$ For aerogels prepared under basic conditions $d_{\mathrm{f}} \approx 1.8$, while for neutral and acidic conditions it is in the range of $2.3-2.4 .^{9,102}$ It is 

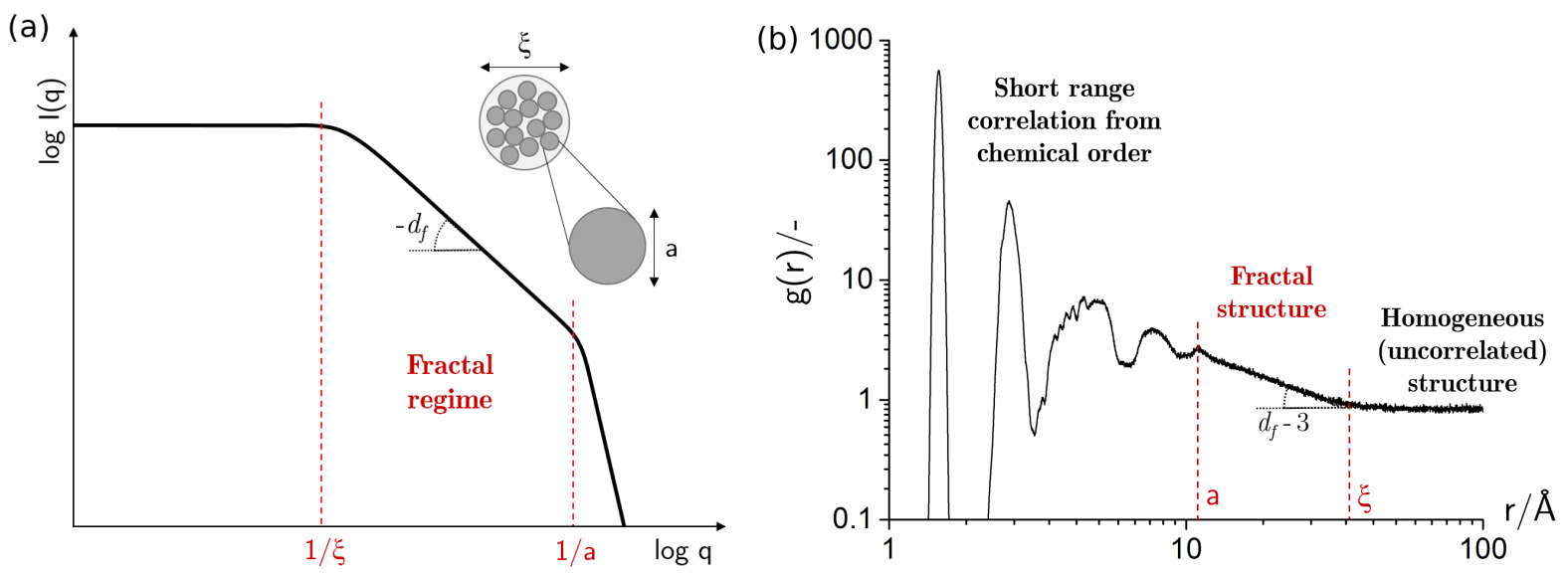

Figure 10: Two methods of estimating the fractal dimension of an aerogel: (a) by the slope of the linear region of a SAXS/SANS spectra, in log-log scale, corresponding to the fractal regime $(1 / \xi<q<1 / a)$ (adapted from Ref. 9); (b) by the slope of the region in the RDF plot (log-log scale) obtained by MD simulations where the peak intensity decays linearly (adapted from Ref. 104)

possible to estimate $d_{\mathrm{f}}$ from MD simulations. For that, the calculated trajectory can be analyzed to extract partial radial distribution functions (RDF) for pairs of atoms $A-B$ $(A, B=\text { Si or } \mathrm{O})^{59}$ :

$$
\left\langle N_{A B}\right\rangle \Delta r=4 \pi r^{2} c_{B} \rho_{N} g_{A B}(r) \Delta r
$$

$\left\langle N_{A B}\right\rangle$ being the average number of $B$-type atoms at a distance between $r$ and $r+\Delta r$ of any atom of $A$, and $\rho_{N}$ the total number density. Then the total RDF, $g(r)$, can be calculated from ${ }^{59}$ :

$$
g(r)=\sum_{A, B} c_{A} c_{B} g_{A B}(r)
$$

where $c_{A}$ and $c_{B}$ are the number concentrations of $A$ and $B$. The plot of $\log (g(r))$ vs. $\log (r)$, as exemplified in Figure 10, exhibits an initial large peak due to short range correlation of chemically bonded atoms in a primary particle. This is followed by a region of correlation where RDF peak intensity decreases linearly, which is characteristic of fractal behavior. As such $d_{\mathrm{f}}$ can be estimated from ${ }^{105}$ :

$$
d_{\mathrm{f}}=3+\frac{\mathrm{d} \log (g(r))}{\mathrm{d} \log (r)}
$$

This region extends up to $r=\xi$, after which $g(r) \rightarrow 1$, indicating a homogeneous noncorrelated structure. 
The creation of an appropriate atomic model for the silica aerogel is crucial to obtain a realistic representation of this fractal structure. DFT calculations can provide minimum energy configurations for silica oligomers up to primary particle size, providing that the FF generated is consistent with these calculations. However, this is insufficient if the goal is to simulate the larger 3D porous structures of the aerogel. To handle this problem, special techniques have been proposed, the most popular being the technique of negative pressure rupturing (NPR), first proposed by Kieffer and Angel ${ }^{106}$, which has been widely used to study various macroscopic properties of TMOS/TEOS-based silica aerogels. It starts with a box of the dense crystalline silica phase $\beta$-crystobalite $\left(\rho=2.2 \mathrm{~g} \mathrm{~cm}^{-3}\right)$, whose atomic structure is well known from X-ray measurements, which is heated to 5000$6000 \mathrm{~K}$, equilibrated, and then quenched to $300 \mathrm{~K}$ to form an amorphous silica structure. The resulting box is then step-wised expanded to the desired density, heated and then quenched to $300 \mathrm{~K}$ (with equilibration periods in between all these procedures). When using the BKS potential an additional 24-6 Lennard-Jones potential needs to be introduced to handle the extreme repulsive forces during the heating/quenching phases. ${ }^{45,46}$ Densities as low as $0.1 \mathrm{~g} \mathrm{~cm}^{-3}$ have been achieved ${ }^{45,105,106}$ with NPR, which are within the typical range of densities exhibited by silica aerogels $\left(0.003-0.35 \mathrm{~g} \mathrm{~cm}^{-311}\right)$, but still leave out interesting aerogel materials corresponding to the range of very low densities $\left(<0.1 \mathrm{~g} \mathrm{~cm}^{-3}\right)$. A modification of the original NPR technique was introduced by Murillo et al. ${ }^{50}$, in which the amorphous box is instantly expanded, heated to $3000 \mathrm{~K}$ and then quenched to $300 \mathrm{~K}$. This instant expansion allows the atoms to diffuse more freely, eventually resulting in a more realistic network of interconnected clusters (although the authors do not report densities below $\left.0.23 \mathrm{~g} \mathrm{~cm}^{-3}\right) .{ }^{50}$ In either version of the NPR technique, the expansion of the box causes the rupturing of several $\mathrm{Si}-\mathrm{O}$ bonds, forming the pores in the structure. The resulting porous network can very well model structural, thermal and mechanical properties of silica aerogels ${ }^{44-50,105,107,108}$. An example of a silica aerogel structure reproduced by this method can be seen in Figure 11. The biggest issue with this procedure is that it does not translate any physical process, as it is merely an artifact used to reproduce the 3D porous network of the aerogels. In other words, it does not mirror in any way the actual sol-gel process. Pohl et al. ${ }^{107}$ pointed out that the $\mathrm{Si} / \mathrm{O}$ proportion 


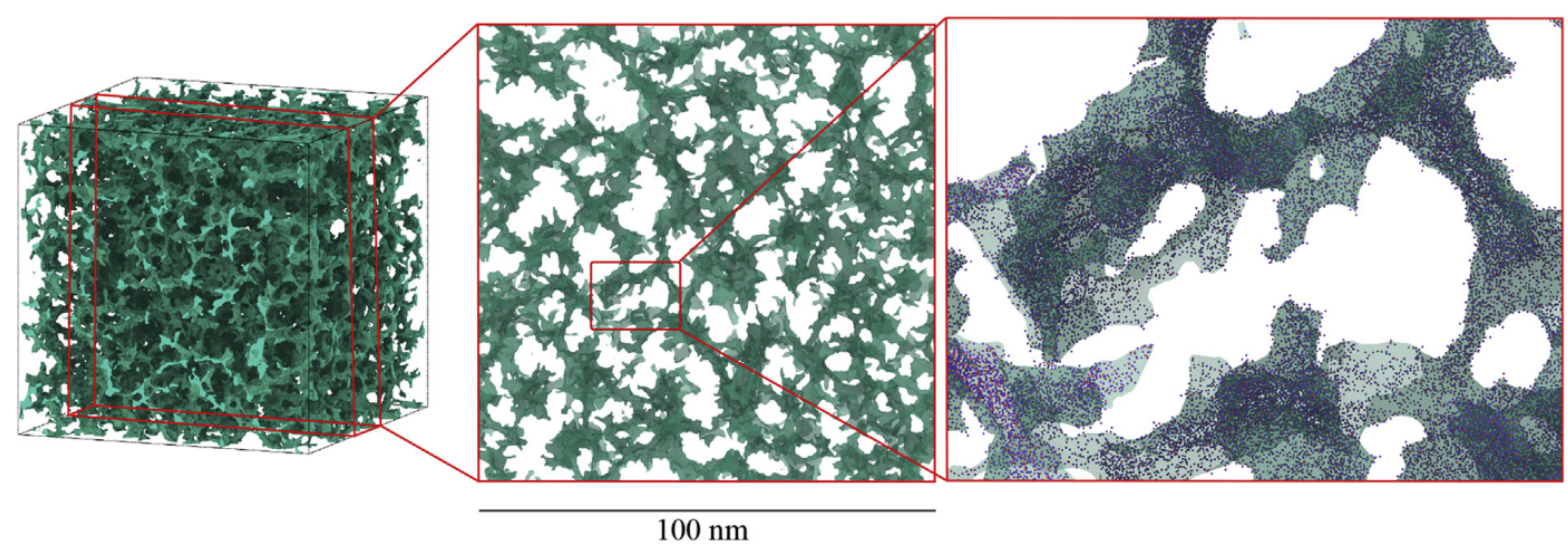

Figure 11: An example of a simulation box $\left(100 \times 100 \times 100 \mathrm{~nm}^{3}\right.$, and more than 7 million atoms) containing a porous silica network generated by the NPR technique, and zoom in images. In the last image, blue and red dots represent $\mathrm{Si}$ and $\mathrm{O}$ atoms, respectively. Reprinted from Acta Materialia, vol. 145, W. Gonçalves, J. Morthomas, P. Chantrenne, M. Perez, G. Foray, and C. L. Martin, "Elasticity and strength of silica aerogels: A molecular dynamics study on large volumes", p. $165-174^{47}$, Copyright (2017), with permission from Elsevier.

of the material generated by this model may deviate from the real proportion because no additional oxygen is added to the system, and suggested a cluster-cluster aggregation approach, in which small clusters of silica are generated with composition obtained from ${ }^{29} \mathrm{Si}-\mathrm{NMR}$ data, randomly assembled together and then bonded by simulating condensation reactions. This procedure reproduces the correct composition and the aerogel fractal structure, but requires a very large amount of clusters to get meaningful results.

Another procedure, named charge rescaling, was proposed by Beckers and de Leeuw ${ }^{104}$, which, tough is still artificial, can reproduce different stages of the sol-gel process, with clear identification of the gel point. The approach consists in spreading free Si and O atoms randomly in the simulation box, with bonding restricted by having the partial charges of the FF reduced to $20 \%$ of the original values. The partial charges are then gradually restored to their original values, promoting bonding between atoms that leads to the final porous network. The gel point can be identified after the charge scaling is done, when a plateau is reached in the plot of the mean squared displacement (MSD) of $\mathrm{Si}$ and $\mathrm{O}$ atoms. The process was proved to be similar to diffusion-limited aggregation, the same mechanism taking place in acid-catalyzed silica condensation. With an additional step of density relaxation by NPT equilibration at ambient pressure - which in this context could be considered analogous to the aging and drying steps of sol-gel synthesis 
- produces a structure with porosity and surface area estimates that are closer to experimental values of comparable materials. However, it should be noted that the relaxation leads to densification (pore colapse), resulting in minimum densities of $0.65 \mathrm{~g} \mathrm{~cm}^{-3}$, which are consistent with silica xerogels (resulting from shrinkage) rather than aerogels. ${ }^{109}$

The NPR technique is generally able to reproduce very well the fractal structure of silica aerogels, with $d_{\mathrm{f}}$ values ranging from 1.8 to 3 , depending on the density of the simulation box. For the bulk densities typical of silica aerogels $\left(<0.35 \mathrm{~g} \mathrm{~cm}^{-311}\right)$ these methods can yield values of $d_{\mathrm{f}}$ well within the experimental range of 1.8-2.4, and practically irrespective of the FF used, as it can be seen in Figure 12. 45,47,50,105,106,108 With the method of Beckers and de Leeuw ${ }^{104}$ the values tend to be slightly higher than those predicted by the NPR method (closer to those of aerogels prepared in acid media). However it is worth mentioning that, in this work, the full representation of the fractal correlation region was limited by the size of the simulation box. ${ }^{104}$ Pore size distributions and surface area can also be estimated by employing algorithms of domain decomposition into cells and classification of cells to determine whether or not they belong to a pore. ${ }^{47,105}$ Pore

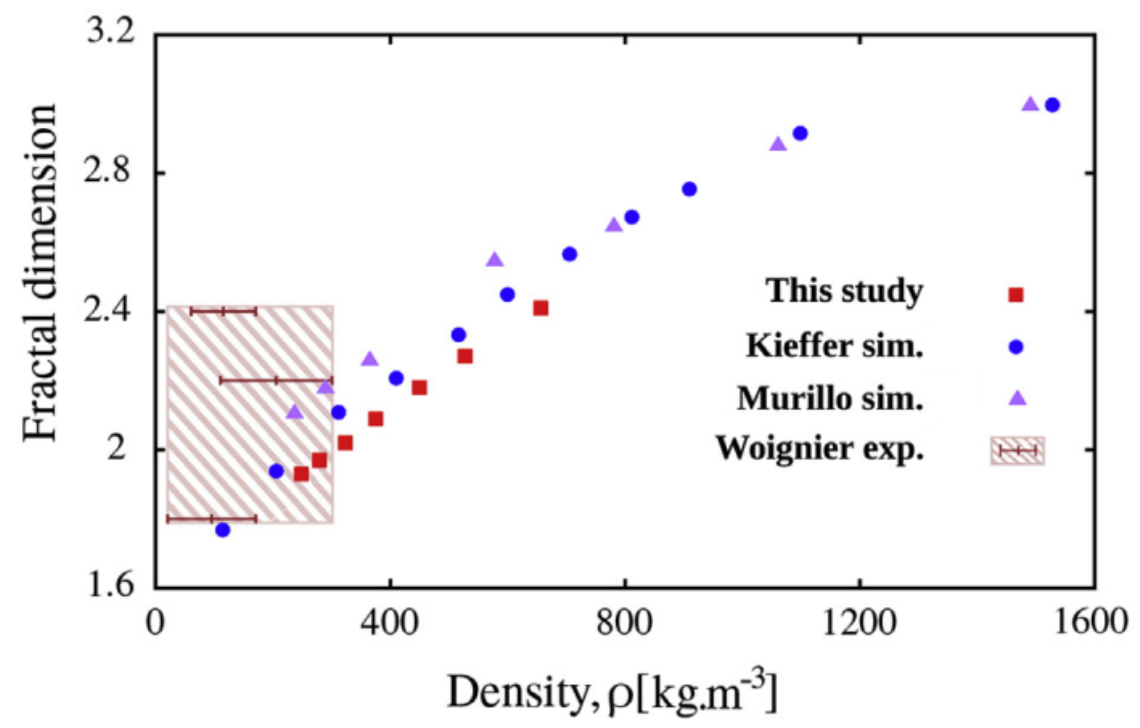

Figure 12: Plot of values of fractal dimension as a function of density, estimated from three different MD simulation studies: Gonçalves et al. ${ }^{47}$, using a modified BKS FF ; Kieffer and Angel ${ }^{106}$ who used a Born-Mayer potential; and Murillo et al. ${ }^{50}$ who used the Vashishta FF. The range of experimental data is also shown, as taken from the work of Woignier et al. ${ }^{102}$ who performed SAXS measurements. Reprinted from Acta Materialia, vol. 145, W. Gonçalves, J. Morthomas, P. Chantrenne, M. Perez, G. Foray, and C. L. Martin, "Elasticity and strength of silica aerogels: A molecular dynamics study on large volumes", p. $165-174^{47}$, Copyright (2017), with permission from Elsevier. 
formation via the NPR method is dependent on the target density, since this determines the degree of box expansion and, therefore, the amount of rupture experienced by the original amorphous network. The result is a Gaussian pore size distribution, whose width was reported ${ }^{47}$ to increase and its center to shift to higher values of pore diameter as density decreases. This effect is represented in Figure 13. For the box formed with the lowest density $\left(0.25 \mathrm{~g} \mathrm{~cm}^{-3}\right)$ a good agreement was also verified between the simulated pore size distribution and its counterpart found for a silica aerogel in an experimental work using electron tomography, for pore sizes up to $25 \mathrm{~nm} .{ }^{47}$ Nakano et al. ${ }^{105}$ reported a power law pore size distribution, but it should be noted that only pores up to $1 \mathrm{~nm}$ were generated in their work, and the simulated system size was orders of magnitude lower than that achieved by Gonçalves et al. ${ }^{47}$ Nevertheless, Nakano et al. ${ }^{105}$ were able to verify that the structural properties of the simulated aerogel were sensitive towards the temperature used in the NPR expansion step.

Even the simpler models with physical FF can give insights on how structural properties are affected by the various reaction conditions. For instance, the ability of surfactants to control pore formation in the aerogel matrix is well documented ${ }^{9}$, and this was verified by Vareda et al. ${ }^{110}$ from a combined experimental-computational study to

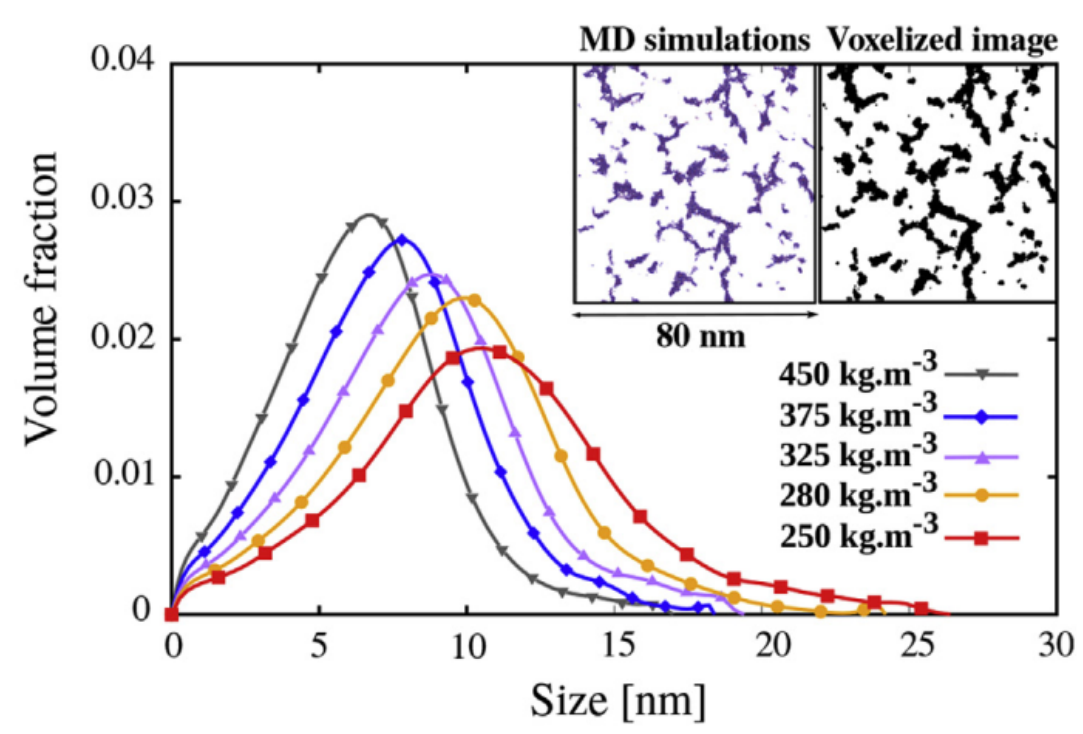

Figure 13: Pore size distribution and its dependency on the system density, generated by the NPR method for a $80 \times 80 \times 80 \mathrm{~nm}^{3}$ simulation box. Reprinted from Acta Materialia, vol. 145, W. Gonçalves, J. Morthomas, P. Chantrenne, M. Perez, G. Foray, and C. L. Martin, "Elasticity and strength of silica aerogels: A molecular dynamics study on large volumes", p. $165-174^{47}$, Copyright (2017), with permission from Elsevier. 
investigate the effect of different surfactants on the structure of MTMS-based silica aerogels. Substantial differences in porosity, average pore size and specific surface area were unveiled depending on the addition of different types of surfactant (anionic: sodium dodecylsulfate, SDS; cationic: cetyltrimtheylammonium bromide, CTAB; neutral: Pluronic F-127). By performing MD simulations, with the GROMOS FF for the anionic and cationic surfactants in a box of silica primary particles and methanol, the authors found that the presence of these species altered the size distribution of aggregates of primary particles. The addition of cationic surfactant in increasing concentrations was found to lead to smaller aggregates, while the anionic surfactant led to larger aggregates at first, but for higher concentrations the average aggregate size was reduced. ${ }^{110}$

Simulations with physical FF can also elucidate the physical interactions that lead up to the sol-gel reactions. By simulating (FF: cff91) a system of TMOS or TEOS in a water/alcohol mixture (pre-hydrolysis system), the hydrolyzed monomers in the respective alcohol (pre-condensation system), and the dimers in water/alcohol mixture (representing the post-condensation conditions), Pereira et al. ${ }^{43}$ were able to demonstrate the physical processes of diffusion that take place before these reactions. The calculated RDFs showed an increased and preferential proximity of water to the Si atom of the precursors along the simulation time in hydrolysis, and a preferential proximity between Si atoms of monomers in the pre-condensation system (at the expense of monomersolvent interactions). On the other hand, they found that solvation by the alcohol became more important after the dimer formation. An analogous study was performed by Jang et al. ${ }^{42}$ with the objective of determining the role of an excess of water molecules in the condensation of $\mathrm{Si}(\mathrm{OH})_{4}$. For that, MD simulations with the CHARMM FF, with parameters for $\mathrm{Si}(\mathrm{OH})_{4}$ obtained from QM calculations, were performed on a system with only $\mathrm{Si}(\mathrm{OH})_{4}$ molecules and another with equal number of $\mathrm{Si}(\mathrm{OH})_{4}$ and water molecules. They concluded that water molecules could disrupt the formation of cyclic-like hydrogen bonded clusters of $\mathrm{Si}(\mathrm{OH})_{4}$, as they occupied the $\mathrm{OH}$ groups in $\mathrm{Si}(\mathrm{OH})_{4}$ with hydrogen bonds, and thus excluded other monomer molecules. While this was only a physical model, it could provide a partial explanation for the effect of increased gelation times with increasing water content (beyond the stoichiometric ratio) reported for acid catalyzed 
condensation. ${ }^{9,42,111,112}$

Reactive FFs allow the direct study of sol-gel reactions at a scale much larger than that attainable by QM methods. Garofalini et al. ${ }^{113}$ studied the early stages of condensation reactions of $\mathrm{Si}(\mathrm{OH})_{4}$ by performing $\mathrm{MD}$ simulations with the reactive FeustonGarofalini potential. The highly condensed silica network was successfully reproduced at the end of the simulations (120 ps). The reactions to form dimers, trimers and tetramers proceeded with the correct mechanism, via the formation of the transition state with a pentacoordinated Si atom leading to the formation of the siloxane bonds. Linear species were predominant at the beginning of the simulation, eventually giving way to cyclic structures (predominantly cyclic tetramers), in agreement with QM results and spectroscopic measurements, as discussed in section 3.1. ${ }^{17,96}$ In fact, the time evolution of the various $\mathrm{Q}^{n}$ Si environments had similar profiles to those observed by NMR, with $\mathrm{Q}^{1}$ and then $\mathrm{Q}^{2}$ appearing at early stages, followed by their consumption with a concomitant increase in $\mathrm{Q}^{3}$, and afterwards $\mathrm{Q}^{4}$. Similar time profiles were obtained from MD simulations of $\mathrm{Si}(\mathrm{OH})_{4}$ /water systems performed by Rao and Gelb ${ }^{114}$, also with the Feuston-Garofalini potential (albeit for a $\sim 3$ times larger system than Garofalini et al. ${ }^{113}$, and simulation times between 1.0 and $12.5 \mathrm{~ns}$ ). By monitoring the size of the formed clusters as the simulation progressed, the authors established two different growth regimes: individual cluster growth by gradual monomer addition, which dominated the early stages of condensation, and cluster-cluster aggregation, which became dominant at the later stages. This change of one regime to the other was clearly visible with water: $\mathrm{Si}(\mathrm{OH})_{4}$ molar ratios (W:M) lower than or equal to 3 , as an abrupt reduction occurred in overall polymerization rate (monomer consumption) very early on, at which point the overall rate became independent of temperature (Figure 14-(a)), an indication of the prevalence of the diffusion limited cluster-cluster aggregation process. On the other hand, for values of $\mathrm{W}: \mathrm{M}>3$, the distinction between both regimes was not so clear, as small clusters steadily grown into larger clusters with a nearly constant polymerization rate, and linear clusters undergone cyclization. While the systems with $\mathrm{W}: \mathrm{M}>3$ could reach the same extent of polymerization as those of $\mathrm{W}: \mathrm{M} \leq 3$, they did so in a much longer time, supporting the idea of longer gel times as well. ${ }^{114}$ Bhattacharya and Kieffer ${ }^{115}$ made a similar evaluation 
(a)

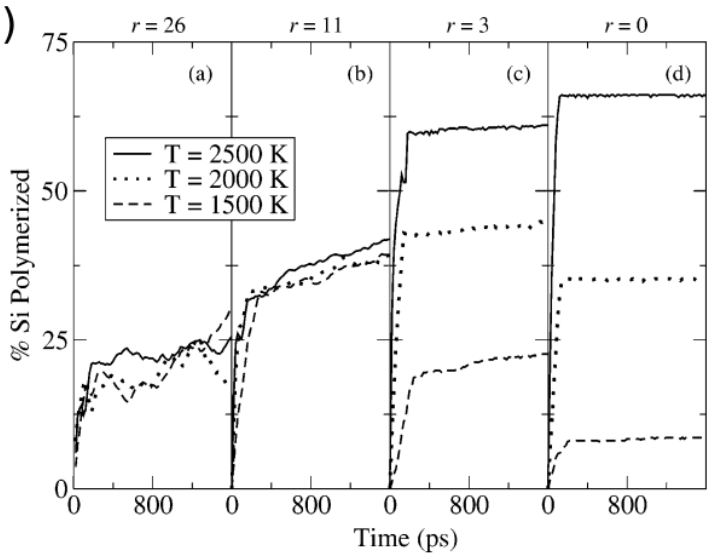

(b)

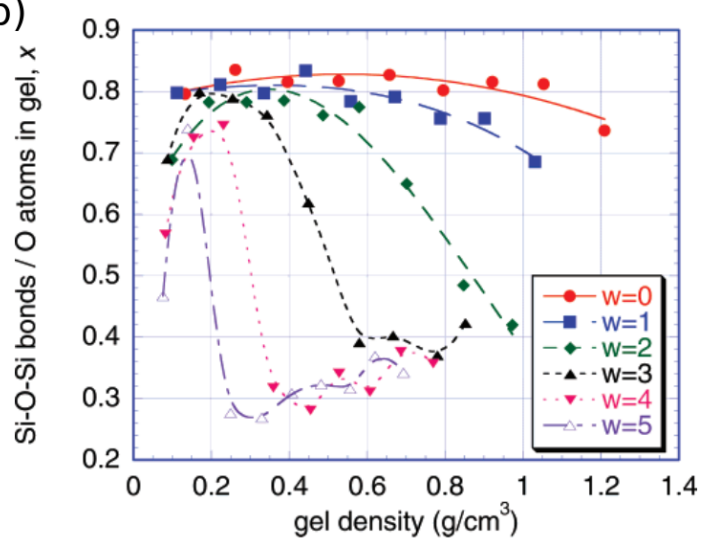

Figure 14: (a) \% Si polymerized as a function of time for $\mathrm{W}: \mathrm{M}$ ratios (here denoted as r) varying between 0 and 26 and also for three different temperatures (1500 to $2500 \mathrm{~K}$ ). Reprinted (adapted) with permission from Rao, N. Z., Gelb, L. D., "Molecular Dynamics Simulations of the Polymerization of Aqueous Silicic Acid and Analysis of the Effects of Concentration on Silica Polymorph Distributions, Growth Mechanisms, and Reaction Kinetics", J. Phys. Chem. B, 2004, 108, 33, 12418-12428 ${ }^{114}$. Copyright (C)2004 American Chemical Society; (b) Average number of siloxane bridges per O atom as a function of density and W:M ratio (named $\mathrm{w}$ in the legend). Reprinted (adapted) with permission from Bhattacharya, S., Kieffer, J., "Molecular Dynamics Simulation Study of Growth Regimes during Polycondensation of Silicic Acid: from Silica Nanoparticles to Porous Gels", J. Phys. Chem. C, 2008, 112, 6, 1764-1771 ${ }^{115}$. Copyright (C)2008 American Chemical Society.

of the effect of $\mathrm{W}: \mathrm{M}$ values on the growth of the silica network, but they also studied the effects of varying the system density using MD simulations with the Feuston-Garofalini potential and starting from a box of $\mathrm{Si}(\mathrm{OH})_{4}$ molecules and water. The simulations, performed over a wide range of conditions, revealed three distinct polymerization behaviours (see Figure 14-(b)):

i) At low densities, and for all values of $\mathrm{W}: \mathrm{M}$, a small number of silica nanoparticles were formed but did not coalesce.

ii) At higher densities and low $\mathrm{W}: \mathrm{M}$ values a continuous $3 \mathrm{D}$ network was formed after aggregation of large clusters, with long range correlations observed in RDF plots and the linear increase in fractal dimension with density, typical of the aerogel fractal structure.

iii) At higher densities and larger $\mathrm{W}: \mathrm{M}$ values $(\mathrm{W}: \mathrm{M}>2)$ the condensation process produced a large number of dispersed branched oligomers (no long range RDF correlations). 
The polymerization process under conditions i) and ii) was found to be limited by monomer diffusion, while under conditions of type iii) the polymerization rate was independent of diffusion coefficients, which means that the process here was not limited by diffusion. The authors also simulated a drying process, by gradual removal of water molecules, and found that the structures produced under the conditions of type ii) presented little density variation, while those prepared in the other types did exhibit a large density increase due collapse.

\subsubsection{Mechanical properties}

MD simulations have been used to study the mechanical properties of silica aerogels. Uniaxial tensile/compression tests can be simulated by stretching/compressing the simulation box along one of its dimensions at a constant strain rate (typical values in the order of 0.0001 to $0.004 \mathrm{ps}^{-1}$ ) in an NPT ensemble. ${ }^{47-50,108}$ The NPR technique was applied in these studies to generate the porous silica network model, with the values of fractal density being used to benchmark the model. The volume of the box and the strain rate were noted to have an effect on the values of Young's modulus, but they converged asymptotically to a constant value for larger boxes and strain rates below $0.004 \mathrm{ps}^{-1} .^{48}$ It is also worth mentioning that larger simulation boxes led to predictions of Young's modulus that were more precise because the sampling of pore size upon the creation of the box was improved. ${ }^{47}$

From experimental tensile tests on silica aerogels it is known that both Young's modulus $(Y)$ and tensile strength $(\sigma)$ increase with bulk density according to a power law $^{9,50}$ :

$$
\begin{aligned}
& Y \propto \rho^{a} \\
& \sigma \propto \rho^{b}
\end{aligned}
$$

The values of $Y$ and $\sigma$ estimated by the MD-generated stress-strain curves, summarized in Table 1, also follow a similar power law with exponents very close to those observed experimentally. The values of $Y$ are also very close to their experimental counterparts in 
Table 1: Summary of Young's modulus and tensile strength power law exponents derived from MD simulations and measured experimentally. Compiled from Refs. 48,50,53.

\begin{tabular}{|c|c|c|c|c|c|}
\hline Works & & $\begin{array}{l}\text { Young's modulus } \\
\text { exponent }(a)\end{array}$ & $\begin{array}{l}\text { Tensile strength } \\
\text { exponent }(b)\end{array}$ & $\begin{array}{l}\text { Range of density } \\
\text { values }\left(\text { g.cm }{ }^{-3}\right)\end{array}$ & Notes \\
\hline \multirow{6}{*}{ Simulation } & 118 & $3.5 \pm 0.2$ & - & $1.67-2.2$ & Vashishta FF, $V_{\text {box }}=46656 \mathrm{~nm}^{3}$ \\
\hline & 50 & $3.11 \pm 0.2$ & $2.53 \pm 0.15 *$ & $0.23-2.2$ & Vashishta FF, $V_{b o x}=2744 \mathrm{~nm}^{3}$ \\
\hline & 108 & $3.17 \pm 0.21$ & - & $0.57-1.23$ & Tersoff FF, $V_{b o x}=3375 \mathrm{~nm}^{3}$ \\
\hline & 48 & $3.25 \pm 0.1$ & $2.79 \pm 0.1 *$ & $0.28-2.08$ & Vashishta FF, $V_{b o x}=21952 \mathrm{~nm}^{3}$ \\
\hline & 49 & $3.2 \pm 0.15$ & - & $0.30-1.16$ & Vashishta FF, $V_{b o x}=151230 \mathrm{~nm}^{3}$ \\
\hline & 47 & $3.84 \pm 0.22 *$ & - & $0.25-0.78$ & Wolf-BKS FF, $V_{b o x}=512000 \mathrm{~nm}^{3}$ \\
\hline \multirow{5}{*}{ Experiment } & 119 & $3.49 \pm 0.07$ & - & $0.14-2.7$ & Pristine silica aerogel \\
\hline & 119 & $2.97 \pm 0.05$ & - & $0.08-1.2$ & Sintered silica aerogel \\
\hline & 116 & $3.8 \pm 0.2$ & - & $0.1-0.4$ & TMOS-based pristine aerogel (neutral pH) \\
\hline & 117 & $3.7 \pm 0.2$ & $2.6 \pm 0.2$ & $0.06-0.5$ & TMOS-based pristine aerogel (all $\mathrm{pH}$ conditions) \\
\hline & 117 & $3.2 \pm 0.2$ & $2.3 \pm 0.2$ & $0.42-2.2$ & Partially densified TMOS-based aerogel \\
\hline
\end{tabular}

* In these cases, even though the exponent was close to the experimental value, the values of Young's modulus or tensile strength were actually systematically deviated from the experimental values.

the same range of densities, although it should be noted that the values of $\sigma$ obtained in MD simulation by Murillo et al. ${ }^{50}$ were 4 times lower than the experimental values (see Figure 15-(b)). The effect of the size of the simulation box on the quality of results is clear when looking at the exponent values predicted by Gonçalves et al. ${ }^{53}$ in Table 1 , which got much closer to the values characteristic of pristine aerogels, while the values of earlier publications could be better compared to sintered/densified silica aerogels.

By calculating the ratio of longitudinal and transversal strain, Patil et al. ${ }^{48}$ could estimate the Poisson ratio of a silica aerogel in the range 0.18 to 0.21 (very close to experimental values, reported in the range 0.205-0.230). Tensile curves for higher den-

(a)

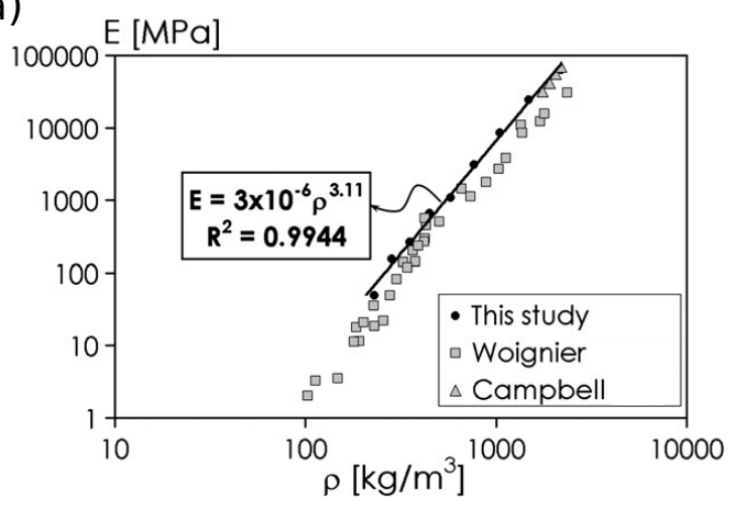

(b)

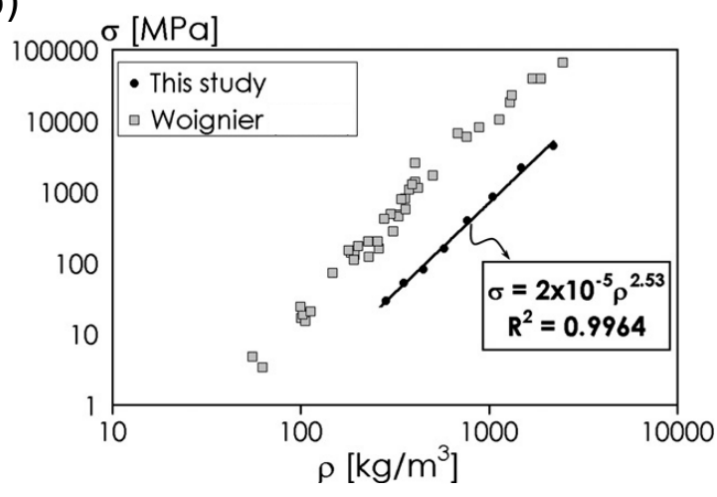

Figure 15: Young's modulus (a) and tensile strength (b) as a function of bulk density. Experimental data (grey squares ${ }^{116,117}$ ) and values from a previous MD study (grey triangles ${ }^{118}$ ) represented for comparison. Reprinted from Journal of Non-Crystalline Solids, vol. 356, Rivas Murillo, J. S, Bachlechner, M. E., Campo, F. A., Barbero, E. J., "Structure and mechanical properties of silica aerogels and xerogels modeled by molecular dynamics simulation", p. 1325 - $1331^{50}$, Copyright (2010), with permission from Elsevier. 
sity exhibited a plastic regime with stress fluctuations (bond rearrangement), followed by yield (due to sudden bond breaking on crack failure). On the other hand, simulated compression stress-strain profiles show three zones, as observed experimentally: i) elastic deformation region, ii) plastic deformation, and iii) densification. The authors also performed cyclic compression simulations (loading followed by unloading) and found hysteretic behavior, with accumulation of a residual strain after unloading in each cycle (Figure $16(\mathrm{a})$ ). This residual strain increased after each cycle of a bigger maximum strain. Ultimately, in the densification region almost no strain recovery existed upon unloading. The stress-strain profile was accompanied by an exponential increase in dissipated energy with maximum strain applied on each cycle. Similarly, hysteresis loops were found in cyclic compression stress-strain curves simulated by Gonçalves et al. ${ }^{47}$ (Figure 16 (b)), using a record-sized box with more than seven million atoms of Si and O (see the box in Figure 11). The simulation of a system of such a size was made possible through the use of the high-performance Wols-BKS FF. ${ }^{53,120}$ Hysteresis was only found for compression cycles, and not for tensile simulations, and such a behavior was greatly reduced
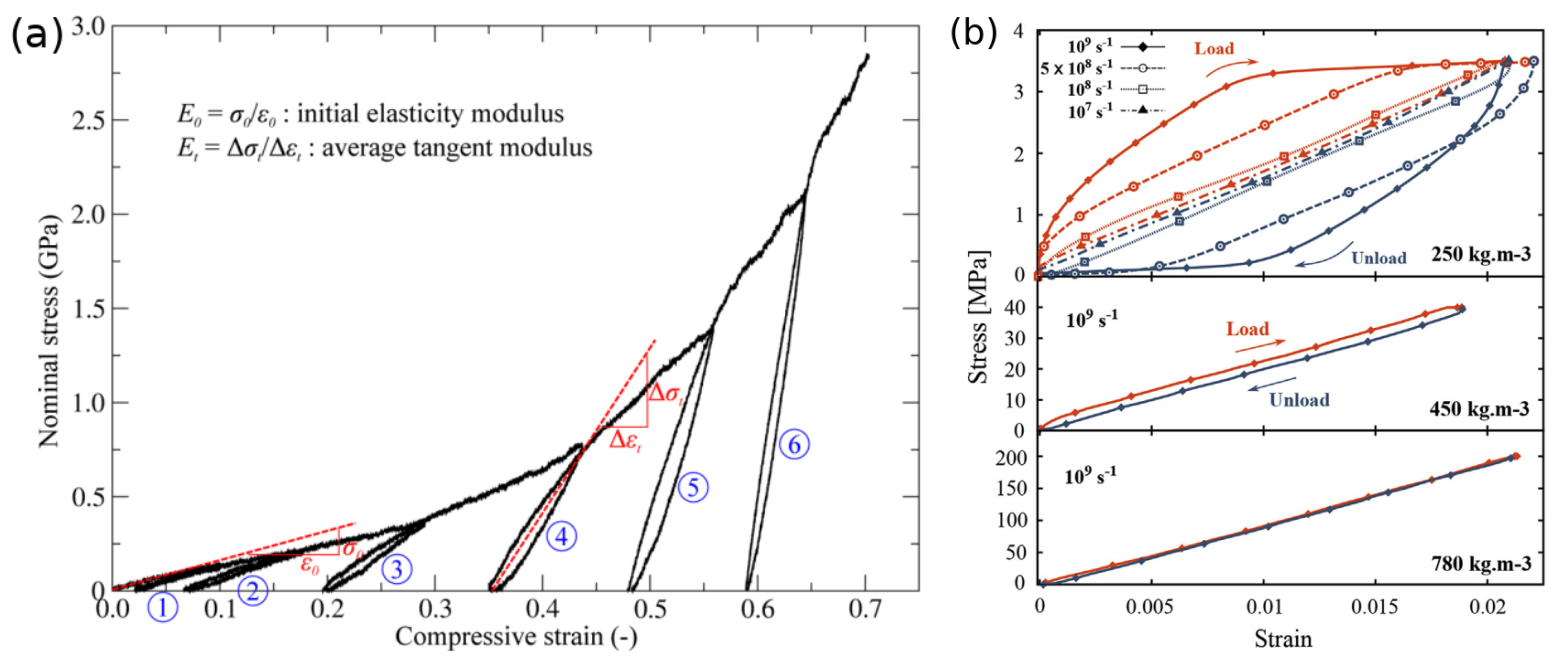

Figure 16: (a) Stress-strain curve of 6 cycles of compressive loading followed by unloading. Reprinted (adapted) with permission from Patil, S. P., Rege, A., Mikhail, Sagardas, Itskov, M., Markert, B., "Mechanics of Nanostructured Porous Silica Aerogel Resulting from Molecular Dynamics Simulations", J. Phys. Chem. B, 2017, 121, 22, 5660-5668 48. Copyright (C) 2017 American Chemical Society; (b) Stress-strain curve of one cycle of compressive loading/unloading, for different densities (top, middle and bottom) and for the lowest density, different values of strain rate (shown in the legend of the top left-hand corner). Reprinted from Acta Materialia, vol. 145, W. Gonçalves, J. Morthomas, P. Chantrenne, M. Perez, G. Foray, and C. L. Martin, "Elasticity and strength of silica aerogels: A molecular dynamics study on large volumes", p. $165-174^{47}$, Copyright (2017), with permission from Elsevier. 
when the aerogel's bulk density increased, as shown in Figure 16 (b). Consequently, the energy loss coefficient, which is proportional to the area of the hysteresis loop, decreased with density concurrently with a decrease in specific surface area, thus suggesting that larger surface areas allow for greater structural rearrangements for energy dissipation upon plastic deformation. Plastic deformation was linked to the observed breaking of "nanoligaments" of silica within the matrix.

Besides tensile and compression tests, other types of mechanical test simulations can give insights on the mechanical behavior of silica aerogels. Patil et al. ${ }^{49}$ simulated, using the Vashishta FF, the propagation of a crack in the silica structure upon transversal tensile strain, starting from two opposite v-shaped notches pre-introduced into the structure. The authors found correlations between the notch length to width ratio and fracture strength (maximum stress before break), fracture toughness (ability of the material to resist crack extension) and energy release rate during fracture. They also found power law relations between the latter two quantities and density. In a later study by Patil et al. ${ }^{121}$ (also with the Vashishta FF), the response of a silica aerogel beam-like box to the propagation of a shock wave was investigated. The shock wave was generated by moving the beam at constant speed towards a fixed reflective wall, and the density spatial and time distribution profiles were used to calculate the shock velocities. For the modeled aerogel of the lowest density $\left(0.209 \mathrm{~g} \mathrm{~cm}^{-3}\right)$ the values matched closely the experimental estimations, and the linear profile of shock velocity vs. initial velocity was also very accurate. The density of the aerogel model had a marked effect on the behavior of the compressed impact region, with lower densities leading to higher dissipated energies and lower von Mises stresses, and also on the propagation of the shock wave which traveled further in the denser materials. Recently, Patil et al. ${ }^{122}$ and Patil ${ }^{123}$ investigated the effects of nanoindentation on pristine silica aerogels and on their composites with graphene (intercalated graphene sheets and silica slabs), respectively. The indentation was simulated by moving a rigid hemispherical diamond tip at constant speed towards the material until a depth of $8-9 \mathrm{~nm}$ was reached, at which point the tip is retracted and the system allowed to relax. The indentation force was recorded along the entire path. In the case of the pristine aerogel systems ${ }^{122}$, the maximum indentation force increased and 
elastic modulus decreased with density. For the lowest density modeled $\left(0.274 \mathrm{~g} \mathrm{~cm}^{-3}\right)$ the values of elastic modulus estimated by the MD simulations were close to experimental values. The authors also proposed a new method for estimating the true contact area between the probe and the aerogel (based on the atoms within the contact region), which is crucial for estimating its hardness. Nevertheless, estimated values of hardness significantly deviated fom experimental values, as the latter were obtained for aerogels whose surface was organically modified. On the other hand, in the aerogel/graphene composite systems ${ }^{123}$ the maximum indentation force was found to be higher for the composite system of a silica aerogel slab in contact with a single graphene $(\mathrm{Gr})$ sheet than for either of these materials alone. Both the Gr sheet and the silica aerogel slab were deformed plastically but, when the number of Gr sheets was increased to 2 or 3, not only did the maximum force increase again but the Gr sheets could recover elastically, even though the aerogel was still permanently deformed. An increase in the thickness of the aerogel slab did not produce a significant decrease in maximum indentation force, showing the benefits of the reinforced composite material. The large number of Si and O atoms in the models featured on the three previously mentioned publications, which ranged between 0.7 and 2.2 million atoms ${ }^{121-123}$, is noteworthy and reflects the current efforts in obtaining silica aerogel models that are more representative of the complex porous network of the real material.

The effects of organic groups attached to the silica network (methyl groups, from MTMS, and ethyl linkers, from 1,2-bis(methyldiethoxysilyl)-ethane, BMDEE) on mechanical properties of the aerogel were assessed by Urata et al. ${ }^{51}$ by first generating the gel matrix from the hydrolyzed precursors from MD simulations with the ReaxFF FF, at $2000 \mathrm{~K}$, with water removal to enhance the rate and extent of polymerization, and then simulating stretching, compression and shear loading. The tensile stress-strain curves of TMOS-based aerogel showed much higher Young's modulus than MTMS and BMDEE in the elastic region, and, in contrast to these two precursors, it showed a significant plastic deformation region (fluctuations of stress) with yield point, which attested the known brittleness of TMOS-based aerogels. The same Young's modulus relation was found for compressive test simulations. On the other hand, cyclic loading/unloading compression 
simulations showed large hysteresis loops with residual stress for the TMOS aerogel, as previously observed by Patil et al. ${ }^{48}$ and Gonçalves et al. ${ }^{47}$ for a material of the same chemical composition, indicative of plastic deformation, while BMDEE showed very good recovery ability with little hysteresis and negligible residual stress. The plastic deformation regime of TMOS was attributed to the breaking of $\mathrm{Si}-\mathrm{O}$ bonds in $\mathrm{Q}^{4}$-coordinated $\mathrm{Si}$ atoms, transforming into $\mathrm{Q}^{3}$, and also changes to $\mathrm{Si}-\mathrm{O}-\mathrm{Si}$ bond angle and oligomer ring size distributions. The same effects were verified for MTMS, but on a much lower extent, and they were practically non-existent for BMDEE. The latter observation can explain the good recovery ability noted above. In a recent publication, Urata et al. extended their study of mechanical properties to aerogels possessing organic polymeric crosslinks, derived from polyvinylpolymethylsiloxane (PVPMS) and polyvinylpolysilsesquioxane (PVPSQ). The same modelling strategy was employed as in the previously mentioned work by these authors. It was concluded that the PVPSQ aerogel model posesses higher tensile and compressive Young's moduli as well as higher shear modulus and energy dissipation than PVPMS, and these values are also more sensitive to the extent of polycondensation for the former material. The authors were able to explain these observations in terms of the structural features of both aerogels: PVPSQ possesses more siloxane bridges per Si atom, evidenced by a larger concentration of $\mathrm{Q}^{3} \mathrm{Si}$, and thus its matrix is inherently more rigid than that of PVPMS. Furthermore, on both PVPMS and PVPSQ aerogel models, the formation of silica rings bridging several polymer chains was noted, but for the materials prepared at a low degree of condensation, the majority of these rings are only attached to one polymer chain. This last observation provided a tentative explanation to the fact that PVPMS and PVPSQ exhibited more flexibility than BMDEE, as these single-chain rings remain intact even during high compression.

\subsubsection{Thermal properties}

Thermal properties of silica aerogels have been studied by performing non equilibrium MD (NEMD) simulations. ${ }^{44-46,124}$ Typically the simulation box can be decomposed into cells, with a temperature gradient applied to the cells on opposite ends, as exemplified in Figure 17. The gradient is enforced periodically throughout the simulations by swapping 

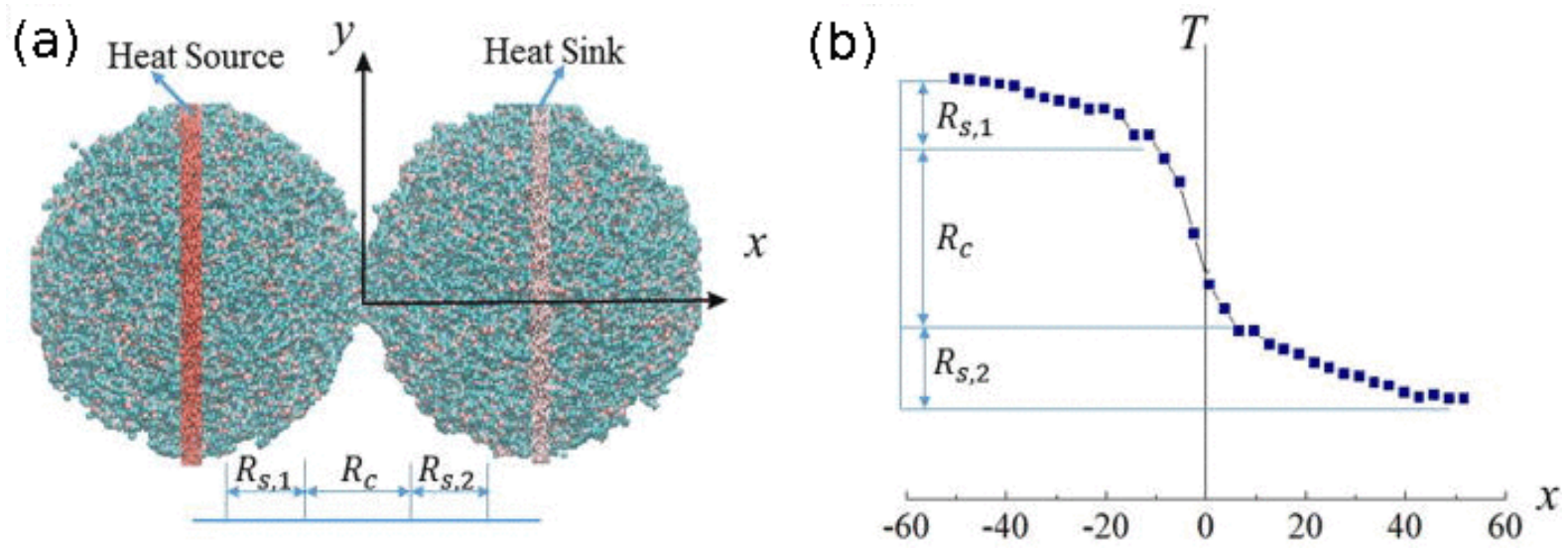

Figure 17: (a) Example of a simulation box for a NEMD simulation of heat flow between two silica secondary particles, where two cells, on the center of each silica particle (diameter $=10 \mathrm{~nm}$ ) are being used as a heat source and sink; (b) the resulting temperature profile along the axial direction. Reprinted from M. Liu, L. Qiu, X. H. Zheng, J. Zhu, and D. W. Tang, "Study on the thermal resistance in secondary particles chain of silica aerogel by molecular dynamics simulation", Journal of Applied Physics, vol. 116, no. 9, p. 093503, 2014 ${ }^{44}$, with the permission of AIP Publishing.

the energies of the atom in the cold cell with highest kinetic energy with the atom of the hot cell with the lowest kinetic energy. The result is a heat flux from the hot to the cold cell across the entire box, thus allowing the thermal conductivity $(\lambda)$ of the material to be estimated. It is widely accepted that thermal conductivity in silica aerogels is the result of three contributions: i) conduction in the solid phase $\left(\lambda_{s}\right)$; ii) conduction in the gas phase $\left(\lambda_{g}\right)$; iii) radiative heat transfer $\left(\lambda_{r}\right)^{9}$ :

$$
\lambda=\lambda_{s}+\lambda_{g}+\lambda_{r}
$$

The relative importance of these contributions depends on the aerogels' structure and composition, but solid phase conduction tends to prevail because of the small size of the pores (5 - $100 \mathrm{~nm}$, being comparable to the mean free path of air molecules), which restricts gas phase conduction by Knudsen flow. ${ }^{9,11}$ Therefore, MD simulations have been focused in obtaining the thermal conductivity of the solid backbone. Ng et al. ${ }^{45}$ first studied this problem by using NEMD simulations (as described above) with a BKS potential and a porous aerogel structure obtained by NPR. They found a power law relationship between solid thermal conductivity and bulk density, a correlation also well known experimentally $^{9,45}$ :

$$
\lambda_{s} \propto \rho^{\alpha}
$$


However, the calculated power law exponent $(\alpha=1.01)$ was closer to the values reported for sintered silica aerogels $(\alpha \approx 1.04)$ when compared to the values of pristine silica aerogels $(\alpha=1.2-2.0)$. This was corrected by Yeo et al. ${ }^{46}$, who performed a similar study but using the re-parameterized Tersoff potential, thus leading to values of thermal conductivity and $\alpha$ closer to experiment $(\alpha=1.61)$. This could be explained by the fact that the simulations using the Tersoff potential could approximate much more closely the experimental vibrational density of states (VDOS) spectra. Nevertheless, a systematic overestimation of the experimental values of $\lambda_{s}$ remained, which was ascribed to limitations in the NPR technique to reproduce some defects, such as micro-cracks and loose-ends in the network, and to produce mesoporosity, as was verified by the computed pore size distributions. ${ }^{45,46}$

Liu et al. ${ }^{44}$ studied the solid heat conduction at a larger scale by simulating a heat flow through the junction of two silica secondary particles both prepared by NPR (see the box in Figure 17), and using a FF based on the re-parameterized Tersoff potential. The authors not only calculated thermal conductivities that were closer to experimental values than the previous two works, with a value of $\alpha$ also inside the range of experimental values, but were also able to conclude that the thermal resistance in the contact region between particles was significantly larger than within the particles. By randomly removing atoms to simulate structural defects, they found that an increase in the concentration of structural defects led to a non-linear increase in thermal resistance. This effect was linked to changes in intensity and frequency of two important bands in the simulated VDOS spectra.

An alternative procedure to NEMD for estimating the thermal conductivity of silica aerogels was recently proposed by Morthomas et al. ${ }^{124}$, aimed at reducing the overall simulation times for large systems. A short NEMD simulation is run, in order to extract the transient temperature profile, and this profile is also estimated by solving the heat equation with an initial estimate of thermal conductivity. A new estimate is then generated by comparing (least squares approach) the temperature profiles from the MD trajectory and the heat equation solution (based on Fourier's Law). This new estimate 
is then used to obtain a new solution to the heat equation, and the process is repeated iteratively until there is convergence in the thermal conductivity values. This methodology was first validated for dense amorphous silica and then used for estimating the thermal conductivity in silica aerogel systems of density $0.25 \mathrm{~g} \mathrm{~cm}^{-3}$ and box volumes up to $90 \times 90 \times 90 \mathrm{~nm}^{3}$, again made possible by employing the Wolf-BKS FF. Slight deviations exists between the MD-generated temperature profiles and those obtained from the heat equation, which arise from kinetic energy fluctuations caused by relaxations during the MD simulation, but it was showed that these have no effect in the final values of thermal conductivity. In spite of the overestimation of the values of thermal conductivity relative to experimental values, which was noted in the validation for dense silica, the obtained density power law exponent for the silica aerogel systems $(\alpha=1.77)$ was even closer to experimental estimates than the value reported by Yeo et al. ${ }^{46}$. The fact that much larger simulation boxes (up to 5 times bigger side length) were used by Morthomas et al. ${ }^{124}$ is certainly the major reason for such better accuracy.

\subsubsection{Surface properties}

In addition to bulk properties MD simulations can also elucidate surface properties of silica aerogels. The surface of unmodified silica aerogels (i.e. derived from TMOS or TEOS) can be considered identical to that of amorphous silica ${ }^{125}$, whose major constituents are siloxane groups and hydroxyl groups. The latter exist with an average concentration of 4.6 to $4.9 \mathrm{OH}$ groups per $\mathrm{nm}^{2}$, as estimated experimentally. ${ }^{126,127}$ Three types of $\mathrm{OH}$ groups have been identified on the surface of amorphous silica: single isolated OH groups, which are lone groups attached to $Q^{3} \mathrm{Si}$ atoms; geminal $\mathrm{OH}$ groups, which are $\mathrm{OH}$ groups attached to the same $Q^{2} \mathrm{Si}$ atom; and vicinal OH groups, which are two neighboring single groups establishing an hydrogen bond between them. ${ }^{128}$ These three configurations are exemplified in Figure 18.

The typical approach to model an amorphous silica surface in MD simulations is to start from a crystalline slab, which is then transformed into an amorphous surface that can be later modified with the desired functional groups. Ceresoli et al. ${ }^{129}$ were 
(a)

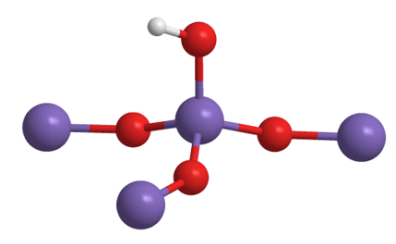

(b)

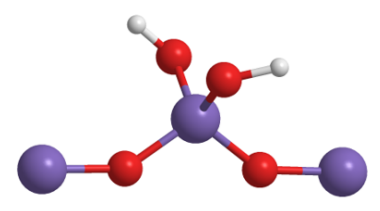

(c)

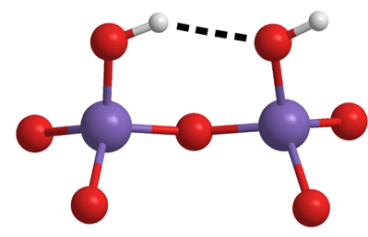

Figure 18: Typical hydroxyl group configurations found on an amorphous silica surface: (a) single hydroxyl groups; (b) geminal hydroxyl groups; (c) vicinal hydroxyl groups. Si atoms in purple, $\mathrm{O}$ atoms in red and $\mathrm{H}$ atoms in white (Color online).

able to reproduce a hydroxylated amorphous silica surface by reconstruction of a (111) $\beta$-cristobalite slab, estimating a hydroxyl concentration of $4.55 \mathrm{OH}$ groups per $\mathrm{nm}^{2}$. Most recently Rimzsa et al. ${ }^{70}$ generated a model of hydroxylated amorphous silica starting from a slab of $\beta$-cristobalite that was transformed into an amorphous and porous structure by iteratively expanding and relaxing the simulation box in the NVT ensemble, followed by NPT relaxation and annealing of the surface to create the non-hydroxylated slab. This surface was then hydroxylated by introducing $\mathrm{OH}$ groups onto defects, such as undercoordinated Si atoms, non-bridging $\mathrm{O}$ atoms and 2-Si rings. The authors used two FF for comparison - ClayFF (a physical FF developed for minerals, including silicates ${ }^{55}$ ) and ReaxFF - and found significant differences between them in terms of: i) distribution of lengths of $\mathrm{Si}-\mathrm{O}$ bonds, although both predicted hydroxylated surfaces to have narrower distributions than the bulk; ii) distribution of Si environments; and iii) concentration of OH groups, with ClayFF getting closer to experimental values. The two FF predicted a decrease in surface energy with increasing hydroxyl concentration but substantially different values of surface energy. While ClayFF predicted energies closer to those observed experimentally during fracture, ReaxFF was in better agreement with the experimental equilibrium surface energies. ${ }^{70}$ It is also worth mentioning here the study of silica aerogel surface properties performed by Gonçalves et al. ${ }^{53}$, who used both the BKS FF and the Wolf-BKS modification to simulate a spherical silica nanoparticle. While the model represented only a non-hydroxylated surface (which experimentally can only be formed at high temperatures ${ }^{125}$ ), it nevertheless gave an indication that the structure of silica near the surface was more heterogeneous than in the core of the particle, especially with regard to the distributions of $\mathrm{Si}-\mathrm{O}-\mathrm{Si}$ and $\mathrm{O}-\mathrm{Si}-\mathrm{O}$ angles and of $\mathrm{Si}$ ring sizes. ${ }^{53}$ 
Reactive FF and/or QM calculations are particularly useful to study complex chemical phenomena taking place at the surface of amorphous silica. Lockwood et al. ${ }^{130}$ carried out a study of proton dynamics at these surfaces by simulating flat silica surfaces and cylindrical pores in contact with water under a reactive silica-water multibody potential, first developed by Mahadevan and Garofalini ${ }^{131}$. This study showed that not only were the lifetimes of hydronium ions shorter close to the surface as compared to bulk water, but also that protonated hydroxyl $\left(\mathrm{SiOH}_{2}{ }^{+}\right)$and siloxane $\left(\mathrm{Si}-\left(\mathrm{OH}^{+}\right)-\mathrm{Si}\right)$ groups were major players in the proton transfer processes occurring at the silica surface, with comparable lifetimes to $\mathrm{H}_{3} \mathrm{O}^{+}$. Furthermore, the results demonstrated that these two species could exist even deeper beneath the surface, particularly $\mathrm{Si}-\left(\mathrm{OH}^{+}\right)-\mathrm{Si}$, and suggested that these groups could transfer protons among themselves due to large scale silica network motions bringing them closer. ${ }^{130}$

Physical FF can also be useful in this context, especially when the aim is to model the interactions of the silica surface with complex molecules. To this end, Cruz-chu et al. ${ }^{56}$ developed a FF specifically to model silica surfaces, called GLASSFF, which is compatible with the popular biomolecular FF CHARMM. The potential energy functions of this FF are in fact analogous to those of INTERFACE FF (in the shape of Equation 6). The authors first constructed amorphous silica surfaces, both hydroxylated and non-hydroxylated (annealed) by employing a reactive FF, and then ran MD simulations with the GLASSFF FF using those surfaces in contact with a water "droplet" (disc). The aim of these simulations was to reproduce the water contact angles on top of the silica surface, so that they could be compared to the experimental values as a mean of parameterizing the $\mathrm{FF}$, particularly the non-bonded interaction parameters. In the end, correct parameter values were found that reliably reproduced the experimental water contact angles for silica surfaces of different hydroxyl group concentrations, up to the known experimental surface concentration. The model was further benchmarked by simulating water permeation through a cylindrical pore. ${ }^{56}$ This FF has been employed, for example, in MD simulations of the interactions of silica surfaces with lipid bilayers ${ }^{132,133}$, graphene $^{134,135}$, salt solutions ${ }^{136}$, and insuline ${ }^{137}$. 
Besides unmodified silica aerogel surfaces, some computational studies regarding other chemical functionalizations have been reported. For instance, Corno et al. ${ }^{52}$ used both DFT calculations (PBE functional and double zeta basis sets) and larger MD simulations with the ReaxFF FF to study hydroxylated silica surfaces functionalized with ethanoic acid or methylamine groups. Both groups engage in hydrogen bonding with neighboring hydroxyl groups, resulting in frequency shifts of their vibrational modes (predicted by DFT), and also impacting their mobility on the surface, with amine groups appearing less mobile than carboxylic groups for concentrations up to $50 \%$. On the other hand, Kanniche et al. ${ }^{138}$ studied not only hydroxylated surfaces, but also ethoxylated (i.e. possessing residual ethoxy groups, that may arise from incomplete hydrolysis) and methylated (in this case two geminal methyl groups), and validated their models by comparing simulated and measured $\mathrm{N}_{2}$ adsorption isotherms. The authors used the BKS FF for the bulk silica, while employing physical FF parameters for the ethoxy and methyl groups that have been derived in other works, and using two $\mathrm{N}_{2} \mathrm{FF}$ for comparison. $\mathrm{N}_{2}$ adsorption simulations at $77 \mathrm{~K}$ showed multilayered adsorption, as exemplified in Figure 19-(a), with calculated isotherms able to match their experimental counterparts very well up to the end of the linear region of the isotherm plot (region of relative pressure less than aprox. 0.6), as seen in Figure 19-(b). Beyond that region the model failed due to the fact that high pressure capillary adsorption could not be accounted for using flat slabs, as explained by the authors. Furthermore, no clear distinctions could be made between the isotherms of the three different functionalized surfaces, as $\mathrm{N}_{2}$ molecules had no selective interactions with any of the functional groups.

\subsection{Mesoscale}

With the CG FF described in section 2.3, Gelb ${ }^{80}$ performed CG-MD simulations of both the gelation process and the aging process of the aerogel. The Langevin equation of motion was used, which differ from regular Newtonian dynamics (Equation 4) by extra stochastic and friction terms (the former simulates Brownian motion and the latter serves to model the effects of the solvent viscosity implicitly) ${ }^{21}$. The simulations of 
(a)

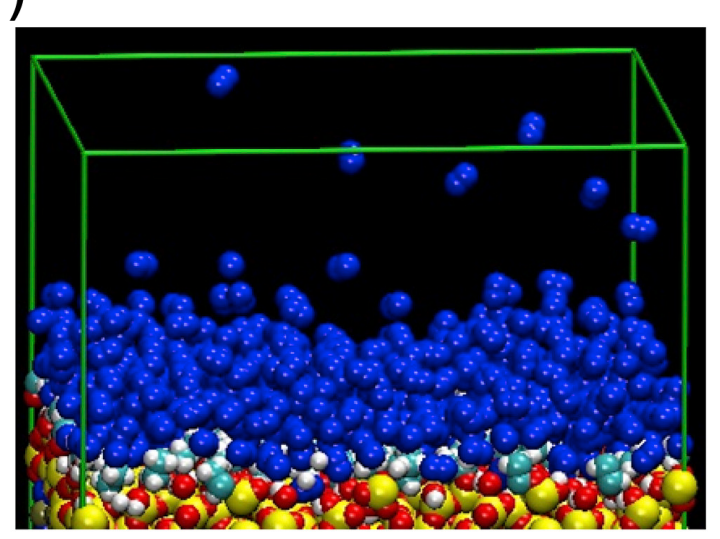

(b)

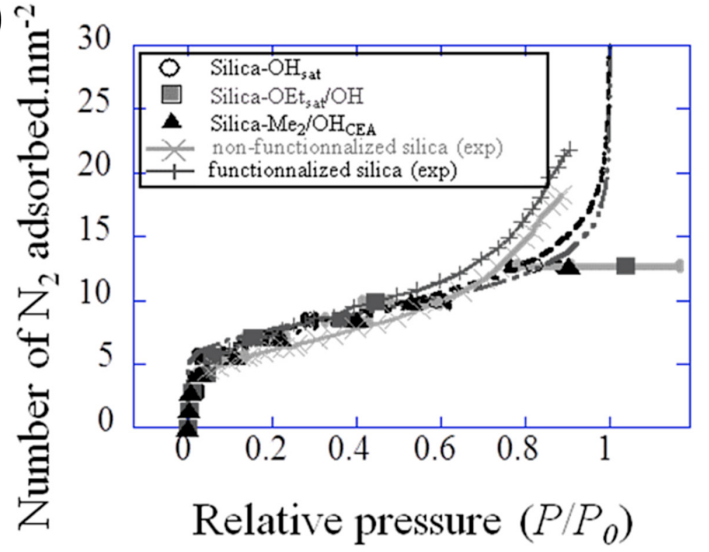

Figure 19: (a) Representative example of an adsorption simulation of $900 \mathrm{~N}_{2}$ molecules onto an amorphous silica surface modified with ethoxy groups (color scheme: Si - yellow, O - red, H - white, C - light blue, N - dark blue); (b) Experimental and calculated $\mathrm{N}_{2}$ adsorption isotherms of the 3 simulated silica surface models. Reprinted from $\mathrm{Mi}$ croporous and Mesoporous Materials, vol. 250, Khanniche,S., Mathieu, D., Pereira, F., Hairault, L., "Atomistic models of hydroxylated, ethoxylated and methylated silica surfaces and nitrogen adsorption isotherms: A molecular dynamics approach", P. $158-169^{138}$, Copyright (2017), with permission from Elsevier.

gelation allowed the identification of the two growth regimes that have also been identified by all-atom MD simulations: an initial cluster growth regime by successive monomer addition, followed by the cluster-cluster aggregation regime, with exponential decrease in the number of clusters with time. Gelb also noticed a strong dependence of the final gel structure with the bond formation probability threshold $(\mathrm{P})$, as lower values of this quantity led to more compact clusters and more porous structures, although lowering beyond a certain point did not result in further changes to the structure, as evidenced by the fractal dimension values becoming constant. Low $\mathrm{P}$ values were identified as the most suitable to approximate experimentally synthesized gels. The Langevin friction coefficient was also found to influence the aerogel structure in a similar manner. The simulation of aging in an NPT ensemble allowed for the relaxation of the structure of the gel, which translated into a slight shrinkage. Aerogels prepared at high $\mathrm{P}$ values tended to exhibit more shrinkage, as the high rate of bond formation did not allow for sufficient relaxation during gelation. However, the predicted values of the bulk modulus were far from the known experimental values for an aerogel of similar density.

This work was continued by Ferreiro-Rangel and Gelb ${ }^{75}$, who employed the same CG model but studied the influence of the particle diameter and volume fraction of particles, 
and, in a later study ${ }^{89}$, performed uniaxial compression/tension simulations to derive the Young's modulus and the Poisson ratio of the aerogels. It is also worth mentioning that these two studies used a much larger system than that of Gelb ${ }^{80}$ (12000 particles vs. 5000), and also simulation times up to $2560 \mathrm{~ns}$ (which was made possible by time steps between 48 and $75 \mathrm{fs}$ ), far more than all-atom MD simulations have achieved, while reaching, at the same time, much lower aerogel densities (as low as $0.06 \mathrm{~g} \mathrm{~cm}^{-3}$ ), which shows, in this case, the advantages of CG-MD in comparison to all-atom MD. In the former study, the authors were able to observe bond formation during the simulations of aging, which was also consistent to what was observed experimentally, and the results suggested that bonds only formed on a local scale. ${ }^{75}$ The shape of the pore size distributions was consistent (and similar to that of Figure 13), regardless of the particle diameter and volume fraction values, which only affected the average pore size. In addition, the bulk modulus values were found to be much closer to the experimental values, and also exhibited the same power law dependence with density, with an exponent of 3.03-3.16, also within the range of the values observed experimentally. ${ }^{75}$ A similar power law relation was found between the bulk modulus and the bond density. In the later studies of Ferreiro-Rangel and Gelb $^{89}$ the mechanical properties of silica aerogels were investigated by the same CG model, using MD simulations as well as hybrid Monte Carlo-molecular dynamics (HMC) simulations. The CG model allowed to capture the previously mentioned power law relationship of the Young's modulus with density, with an exponent of 3.00-3.05 found by the HMC method, and 2.8-2.9 with the MD-only approach, largely independent of particle diameter. These values were closer to the experimental exponents found for sintered aerogels but in a similar range of densities, unlike those obtained from all-atom MD works of Patil et al. ${ }^{48,49}$, Lei et al. ${ }^{108}$ and Murillo et al. ${ }^{50}$, summarized in Table 1. Values of Poisson ratio were found in the interval $0.17-0.24$, which was both close to the experimental values and the previously presented values of Patil et al. using all-atom MD simulations, as well as better than the finite element studies. ${ }^{48,89}$ The authors also carried out uniaxial compression and tensile test simulations under large strains, and found that pore collapse occurred during compression, while under tension the existence of strands of particles supporting the load became evident (Figure 20-(a)). These strands 
(a)

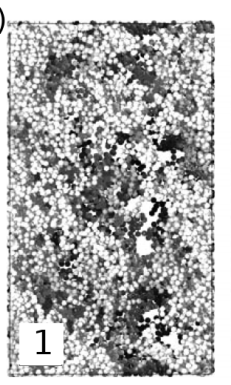

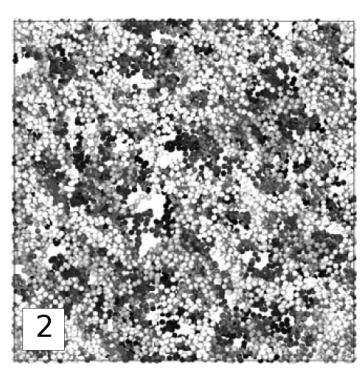
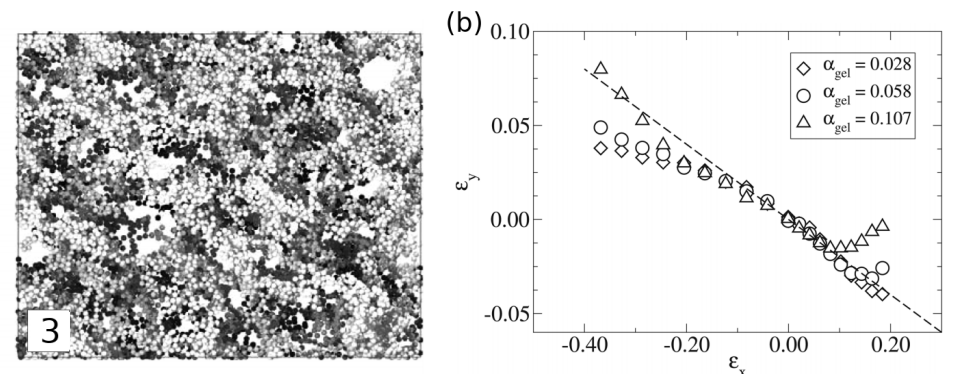

Figure 20: (a) CG silica aerogel network after $40 \%$ compression strain (1), with no strain (2) and after $20 \%$ tensile strain (3), with darker colors representing deeper particles; (b) Plot of transversal vs. axial strain for aerogels of 3 different densities (here represented by volume fraction of particles). The slope of the linear region gives the Poisson ratio. Reprinted (adapted) with permission from Ferreiro-Rangel, C. A., Gelb, L. D., "Computational Study of Uniaxial Deformations in Silica Aerogel Using a Coarse-Grained Model", J. Phys. Chem. B, 2015, 119, 27, 8640-8650 ${ }^{89}$. Copyright (C)2015 American Chemical Society.

may be analogous to the nanoligaments identified by Gonçalves et al. ${ }^{47}$ The density of the gel had an impact on the shape of the stress-strain curves for compression and tension, with denser gels possessing a smaller elastic region and exhibiting failure under tension. The maximum stress and the corresponding value of strain were both found to vary with density by a power law relationship. In addition, denser gels possessed stronger deviations outside of the linear region of the transversal vs. axial strain curves than less dense gels, and even exhibited auxetic behavior (i.e. negative effective Poisson ratios) under tension, as represented in Figure 20-(b). ${ }^{89}$

\section{Conclusions}

MM\&S methods have proven to be extremely useful for studying the complex phenomena taking place during the sol-gel process, something which experiments alone cannot fully accomplish. QM calculations provided crucial evidence to determine the most probable mechanisms taking place in hydrolysis and condensation reactions, out of the various (often conflicting) mechanisms proposed by experimental evidence. Solvent effects on these reactions, such as the direct participation of hydrogen bonded water complexes in some of them, have been studied consistently. However, the strong dependence of the calculated energies and mechanistic details on the type of solvent model used - implicit, 
explicit of both - is a major concern that needs to be assessed judiciously. In this regard, hybrid quantum mechanics/molecular mechanics (QM/MM) MD calculations could also be a viable and reliable method to handle solvent effects, as they allow the silica-based species and their first solvation shell to be treated by QM-based models, while describing the remainder of the solvent by inexpensive classical MD FFs. ${ }^{139}$ To the best of our knowledge, this kind of approach has remained unexplored in the field of MM\&S of solgel chemistry. QM calculations have also been indispensable in identifying the most predominant species at the initial stages of the sol-gel process, through calculation of spectroscopic properties of these particles - IR, Raman, ${ }^{29} \mathrm{Si}-\mathrm{NMR}$ and INS spectra and comparison with the spectra obtained experimentally. This information also has allowed the development of kinetic models for these earlier stages of silica primary particle formation, which reproduce relatively well the profiles obtained experimentally.

At the level of secondary particles, classical MD simulations employing reactive FF and techniques for generation of the porous silica network, such as the popular NPR method, have permitted the estimation of structural, mechanical and thermal properties with very good accuracy. In particular, the known fractal structure of silica aerogels at this scale can be emulated extremely well, with calculated values of fractal dimensions well within experimental values. However, the minimum bulk density achieved by NPR and related techniques still remains limited to $0.1 \mathrm{~g} \mathrm{~cm}^{-3}$. Reactive FF-based simulations can also produce the porous network of silica aerogels, but have revealed more valuable insights into the mass transfer phenomena that regulate its formation - a combination of monomer addition to form clusters and later cluster-cluster aggregation. The effects of water:monomer ratio, temperature and simulated density on these regimes have been thoroughly assessed. MD simulations have also proven to be reliable in estimating both mechanical and thermal properties of silica aerogels, both qualitatively, and, to a great extent, quantitatively. More than just approximating experimental results, MD simulations provided structure-property relations by examining the fundamental phenomena that ultimately lead to such mechanical and thermal properties. More recent works from the last few years, by a combination of powerful hardware and simplified FFs, have successfully tackled all-atom systems in the order of millions of atoms, providing nanoporous 
models of silica aerogels that are closer to reality than ever before.

Finally, coarse-graining has allowed the extension of the realm of MD simulations to the mesoscale through the study of aerogels of lower density, larger size and for longer times than all-atom simulations, with comparative predictive performance in terms of structural and mechanical properties.

MM\&S tools will certainly continue to play a critical role in the design of silica aerogels and related materials, as researchers continue to have increasingly easier access to better and more diverse high-performance computer resources. As a consequence of this enhancement in computing power, it is expected that both QM and MD calculations will be able to tackle even larger systems and larger time scales, which can only lead to results of improved quality. Even though most of the literature available on MM\&S of silica aerogels pertains to TMOS/TEOS-based materials, it is likely that the field will focus more and more on ORMOSIL aerogels and composite materials based on them, much like what has happened historically in the laboratory. The greatest challenge in this endeavor will likely be in the development of new models, particularly FFs, that are able to describe the silica and organic moieties simultaneously.

\section{Acknowledgement}

This work was developed under the project AerogelDustFree - "Aerogel for Space applications ISO8 without dust release" (Contract No 17815), developed by the consortium Active Aerogels / University of Coimbra, funded by the European Regional Development Fund (FEDER) through the Operational Program for Competitiveness and Internationalization and/or the Regional Operational Program of the Center of Portugal (Centro2020) of PT2020. 


\section{References}

(1) Fermeglia, M.; Pricl, S. Multiscale molecular modeling in nanostructured material design and process system engineering. Computers \& Chemical Engineering 2009, 33, 1701 - 1710, Selected Papers from the 18th European Symposium on Computer Aided Process Engineering (ESCAPE-18).

(2) Tramontano, A. The role of molecular modelling in biomedical research. FEBS Letters 2006, 580, 2928 - 2934, Istanbul Special Issue.

(3) Goldbeck, G. The economic impact of molecular modelling of chemicals and materials; 2012.

(4) Aspuru-Guzik, A.; Lindh, R.; Reiher, M. The Matter Simulation (R)evolution. ACS Central Science 2018, 4, 144-152.

(5) de Pablo, J. J.; Escobedo, F. A. Molecular simulations in chemical engineering: Present and future. AIChE Journal 2002, 48, 2716-2721.

(6) Palmer, J. C.; Debenedetti, P. G. Recent advances in molecular simulation: A chemical engineering perspective. AIChE Journal 2015, 61, 370-383.

(7) Wei, J.; Denn, M.; Seinfeld, J.; Chakraborty, A.; Ying, J.; Peppas, N.; Stephanopoulos, G. Molecular Modeling and Theory in Chemical Engineering; Advances in Chemical Engineering; Elsevier Science, 2001.

(8) Weiß, H.; Deglmann, P.; in 't Veld, P. J.; Cetinkaya, M.; Schreiner, E. Multiscale Materials Modeling in an Industrial Environment. Annual Review of Chemical and Biomolecular Engineering 2016, 7, 65-86.

(9) Aegerter, M.; Leventis, N.; Koebel, M. Aerogels Handbook; Advances in Sol-Gel Derived Materials and Technologies; Springer New York, 2011.

(10) Gurav, J. L.; Jung, I.-K.; Park, H.-H.; Kang, E. S.; Nadargi, D. Y. Silica Aerogel: Synthesis and Applications. Journal of Nanomaterials 2010, 2010, 11. 
(11) Vareda, J. P.; Lamy-Mendes, A.; Durães, L. A reconsideration on the definition of the term aerogel based on current drying trends. Microporous and Mesoporous Materials 2018, 258, $211-216$.

(12) Maleki, H.; Durães, L.; Portugal, A. An overview on silica aerogels synthesis and different mechanical reinforcing strategies. Journal of Non-Crystalline Solids 2014, $385,55-74$.

(13) Brinker, C. Hydrolysis and condensation of silicates: Effects on structure. Journal of Non-Crystalline Solids 1988, 100, 31 - 50, Glasses and Glass Ceramics from Gels.

(14) Lamy-Mendes, A.; Silva, R.; Durães, L. Advances in carbon nanostructure-silica aerogel composites: a review. J. Mater. Chem. A 2018, 6, 1340-1369.

(15) Dorcheh, A. S.; Abbasi, M. Silica aerogel: synthesis, properties and characterization. Journal of Materials Processing Technology 2008, 199, 10 - 26.

(16) Borba, A.; Vareda, J. P.; Durães, L.; Portugal, A.; Simões, P. N. Spectroscopic characterization of silica aerogels prepared using several precursors - effect on the formation of molecular clusters. New J. Chem. 2017, 41, 6742-6759.

(17) Depla, A.; Lesthaeghe, D.; van Erp, T. S.; Aerts, A.; Houthoofd, K.; Fan, F.; Li, C.; Van Speybroeck, V.; Waroquier, M.; Kirschhock, C. E. A.; Martens, J. A. ${ }^{29} \mathrm{Si}$ NMR and UV-Raman Investigation of Initial Oligomerization Reaction Pathways in Acid-Catalyzed Silica Sol-Gel Chemistry. The Journal of Physical Chemistry C 2011, 115, 3562-3571.

(18) Al-Oweini, R.; El-Rassy, H. Synthesis and characterization by FTIR spectroscopy of silica aerogels prepared using several $\mathrm{Si}(\mathrm{OR})_{4}$ and $\mathrm{R}^{\prime \prime} \mathrm{Si}\left(\mathrm{OR}^{\prime}\right)_{3}$ precursors. Journal of Molecular Structure 2009, 919, 140 - 145.

(19) Cramer, C. Essentials of Computational Chemistry: Theories and Models, 2nd ed.; Wiley, 2005. 
(20) Nagy, B.; Jensen, F. Reviews in Computational Chemistry; John Wiley \& Sons, Ltd, 2017; Chapter 3, pp 93-149.

(21) Jensen, F. Introduction to Computational Chemistry, 2nd ed.; Wiley, 2007.

(22) Cheng, X.; Chen, D.; Liu, Y. Mechanisms of Silicon Alkoxide HydrolysisOligomerization Reactions: A DFT Investigation. ChemPhysChem 2012, 13, 23922404.

(23) Fernandez, L.; Tuñón, I.; Latorre, J.; Guillem, C.; Beltrán, A.; Amorós, P. Tetraethylorthosilicate as molecular precursor to the formation of amorphous silica networks. A DFT-SCRF study of the base catalyzed hydrolysis. Journal of Molecular Modeling 2012, 18, 3301-3310.

(24) Okumoto, S.; Fujita, N.; Yamabe, S. Theoretical Study of Hydrolysis and Condensation of Silicon Alkoxides. The Journal of Physical Chemistry A 1998, 102, $3991-3998$.

(25) Cheng, X.; He, G.; Li, L.; Liu, Y. Methanolysis of $\mathrm{Si}\left(\mathrm{OCH}_{3}\right)_{4}$ and $\mathrm{Al}\left(\mathrm{OCH}_{3}\right)_{3}$ alkoxides: A comparative study with DFT method. Computational and Theoretical Chemistry 2013, 1023, $19-23$.

(26) Henschel, H.; Schneider, A. M.; Prosenc, M. H. Initial Steps of the Sol-Gel Process: Modeling Silicate Condensation in Basic Medium. Chemistry of Materials 2010, 22, 5105-5111.

(27) McIntosh, G. J. A theoretical kinetic model of the temperature and pH dependent dimerization of orthosilicic acid in aqueous solution. Phys. Chem. Chem. Phys. 2012, 14, 996-1013.

(28) Cypryk, M.; Apeloig, Y. Mechanism of the Acid-Catalyzed Si-O Bond Cleavage in Siloxanes and Siloxanols. A Theoretical Study. Organometallics 2002, 21, 21652175.

(29) McIntosh, G. J. Theoretical investigations into the nucleation of silica growth in 
basic solution part I - ab Initio studies of the formation of trimers and tetramers. Phys. Chem. Chem. Phys. 2013, 15, 3155-3172.

(30) Marx, D.; Hutter, J. Ab Initio Molecular Dynamics: Basic Theory and Advanced Methods; Cambridge University Press, 2009.

(31) Schwerdtfeger, P. The Pseudopotential Approximation in Electronic Structure Theory. ChemPhysChem 2011, 12, 3143-3155.

(32) Handy, N. C. Density Functional Theory. Quantum Mechanical Simulation Methods for Studying Biological Systems. Berlin, Heidelberg, 1996; pp 1-35.

(33) Koch, W. A Chemists Guide to Density Functional Theory, 3rd ed.; Wiley-VCH Verlag GmbH, 2008.

(34) Becke, A. D. Perspective: Fifty years of density-functional theory in chemical physics. The Journal of Chemical Physics 2014, 140, 18A301.

(35) Sousa, S. F.; Fernandes, P. A.; Ramos, M. J. General Performance of Density Functionals. The Journal of Physical Chemistry A 2007, 111, 10439-10452.

(36) Ghosh, S.; Verma, P.; Cramer, C. J.; Gagliardi, L.; Truhlar, D. G. Combining Wave Function Methods with Density Functional Theory for Excited States. Chemical Reviews 2018, 118, 7249-7292.

(37) Yu, H. S.; Li, S. L.; Truhlar, D. G. Perspective: Kohn-Sham density functional theory descending a staircase. The Journal of Chemical Physics 2016, 145, 130901.

(38) Corminboeuf, C.; Tran, F.; Weber, J. The role of density functional theory in chemistry: Some historical landmarks and applications to zeolites. Journal of Molecular Structure: THEOCHEM 2006, 762, 1 - 7, A Collection of Papers Dedicated to Professor Annick Goursot on the Occasion of her 60th Birthday.

(39) Kirste, B. Applications of Density Functional Theory to Theoretical Organic Chemistry. Chemical Sciences Journal 2016, 7, 1-5. 
(40) Tuckerman, M. E. Ab initio molecular dynamics: basic concepts, current trends and novel applications. Journal of Physics: Condensed Matter 2002, 14, 12971355.

(41) Leimkuhler, B.; Matthews, C. Molecular Dynamics: With Deterministic and Stochastic Numerical Methods; Interdisciplinary Applied Mathematics; Springer International Publishing, 2015.

(42) Jang, K.-W.; Pyun, S.-I.; Jhon, M.-S. The Role of Excess Water in Acidic Sol-Gel Polymerization of Tetraethoxysilane (TEOS) Using Molecular Dynamics Simulation. Molecular Simulation 2003, 29, 489-494.

(43) Pereira, J. C. G.; Catlow, C. R. A.; Price, G. D. Molecular Dynamics Simulation of Methanolic and Ethanolic Silica-Based Sol-Gel Solutions at Ambient Temperature and Pressure. The Journal of Physical Chemistry A 2002, 106, 130-148.

(44) Liu, M.; Qiu, L.; Zheng, X. H.; Zhu, J.; Tang, D. W. Study on the thermal resistance in secondary particles chain of silica aerogel by molecular dynamics simulation. Journal of Applied Physics 2014, 116, 093503.

(45) Ng, T.; Yeo, J.; Liu, Z. A molecular dynamics study of the thermal conductivity of nanoporous silica aerogel, obtained through negative pressure rupturing. Journal of Non-Crystalline Solids 2012, 358, 1350 - 1355.

(46) Yeo, J. J.; Liu, Z. S.; Ng, T. Y. Enhanced thermal characterization of silica aerogels through molecular dynamics simulation. Modelling and Simulation in Materials Science and Engineering 2013, 21, 075004.

(47) Gonçalves, W.; Morthomas, J.; Chantrenne, P.; Perez, M.; Foray, G.; Martin, C. L. Elasticity and strength of silica aerogels: A molecular dynamics study on large volumes. Acta Materialia 2018, 145, 165 - 174 .

(48) Patil, S. P.; Rege, A.; Sagardas,; Itskov, M.; Markert, B. Mechanics of Nanostructured Porous Silica Aerogel Resulting from Molecular Dynamics Simulations. The Journal of Physical Chemistry B 2017, 121, 5660-5668, PMID: 28556665. 
(49) Patil, S. P.; Rege, A.; Itskov, M.; Markert, B. Fracture of silica aerogels: An allatom simulation study. Journal of Non-Crystalline Solids 2018, 498, 125 - 129.

(50) Murillo, J. S. R.; Bachlechner, M. E.; Campo, F. A.; Barbero, E. J. Structure and mechanical properties of silica aerogels and xerogels modeled by molecular dynamics simulation. Journal of Non-Crystalline Solids 2010, 356, 1325 - 1331.

(51) Urata, S.; Kuo, A.-T.; Murofushi, H. Origin of Flexibility of Organic-Inorganic Aerogels: Insights from Atomistic Simulations. The Journal of Physical Chemistry C 2018, 122, 20555-20563.

(52) Corno, M.; Delle Piane, M.; Monti, S.; Moreno-Couranjou, M.; Choquet, P.; Ugliengo, P. Computational Study of Acidic and Basic Functionalized Crystalline Silica Surfaces as a Model for Biomaterial Interfaces. Langmuir 2015, 31, 63216331, PMID: 26010674.

(53) Gonçalves, W.; Morthomas, J.; Chantrenne, P.; Perez, M.; Foray, G.; Martin, C. L. Molecular dynamics simulations of amorphous silica surface properties with truncated Coulomb interactions. Journal of Non-Crystalline Solids 2016, 447, 1 - 8.

(54) Heinz, H.; Lin, T.-J.; Kishore Mishra, R.; Emami, F. S. Thermodynamically Consistent Force Fields for the Assembly of Inorganic, Organic, and Biological Nanostructures: The INTERFACE Force Field. Langmuir 2013, 29, 1754-1765, PMID: 23276161.

(55) Cygan, R. T.; Liang, J.-J.; Kalinichev, A. G. Molecular Models of Hydroxide, Oxyhydroxide, and Clay Phases and the Development of a General Force Field. The Journal of Physical Chemistry B 2004, 108, 1255-1266.

(56) Cruz-Chu, E. R.; Aksimentiev, A.; Schulten, K. Water-Silica Force Field for Simulating Nanodevices. The Journal of Physical Chemistry B 2006, 110, 21497-21508.

(57) Feuston, B. P.; Garofalini, S. H. Empirical three-body potential for vitreous silica. The Journal of Chemical Physics 1988, 89, 5818-5824. 
(58) Feuston, B.; Garofalini, S. Onset of polymerization in silica sols. Chemical Physics Letters 1990, 170, $264-270$.

(59) Vashishta, P.; Kalia, R. K.; Rino, J. P.; Ebbsjö, I. Interaction potential for $\mathrm{SiO}_{2}$ : A molecular-dynamics study of structural correlations. Phys. Rev. B 1990, 41, $12197-12209$.

(60) van Beest, B. W. H.; Kramer, G. J.; van Santen, R. A. Force fields for silicas and aluminophosphates based on ab initio calculations. Phys. Rev. Lett. 1990, 64, $1955-1958$.

(61) Carré, A.; Horbach, J.; Ispas, S.; Kob, W. New fitting scheme to obtain effective potential from Car-Parrinello molecular-dynamics simulations: Application to silica. EPL (Europhysics Letters) 2008, 82, 17001.

(62) Carré, A.; Berthier, L.; Horbach, J.; Ispas, S.; Kob, W. Amorphous silica modeled with truncated and screened Coulomb interactions: A molecular dynamics simulation study. The Journal of Chemical Physics 2007, 127, 114512.

(63) Munetoh, S.; Motooka, T.; Moriguchi, K.; Shintani, A. Interatomic potential for SiO systems using Tersoff parameterization. Computational Materials Science 2007, 39, $334-339$.

(64) van Duin, A. C. T.; Strachan, A.; Stewman, S.; Zhang, Q.; Xu, X.; Goddard, W. A. ReaxFFSiO Reactive Force Field for Silicon and Silicon Oxide Systems. The Journal of Physical Chemistry A 2003, 107, 3803-3811.

(65) Mortier, W. J.; Ghosh, S. K.; Shankar, S. Electronegativity-equalization method for the calculation of atomic charges in molecules. Journal of the American Chemical Society 1986, 108, 4315-4320.

(66) Fogarty, J. C.; Aktulga, H. M.; Grama, A. Y.; van Duin, A. C. T.; Pandit, S. A. A reactive molecular dynamics simulation of the silica-water interface. The Journal of Chemical Physics 2010, 132, 174704. 
(67) Moqadam, M.; Riccardi, E.; Trinh, T. T.; Åstrand, P.-O.; van Erp, T. S. A test on reactive force fields for the study of silica dimerization reactions. The Journal of Chemical Physics 2015, 143, 184113.

(68) Rimsza, J. M.; Yeon, J.; van Duin, A. C. T.; Du, J. Water Interactions with Nanoporous Silica: Comparison of ReaxFF and ab Initio based Molecular Dynamics Simulations. The Journal of Physical Chemistry C 2016, 120, 24803-24816.

(69) Rimsza, J. M.; Du, J. Interfacial Structure and Evolution of the Water-Silica Gel System by Reactive Force-Field-Based Molecular Dynamics Simulations. The Journal of Physical Chemistry C 2017, 121, 11534-11543.

(70) Rimsza, J. M.; Jones, R. E.; Criscenti, L. J. Surface Structure and Stability of Partially Hydroxylated Silica Surfaces. Langmuir 2017, 33, 3882-3891, PMID: 28375622.

(71) Yeo, J.; Liu, Z.; Ng, T. Y. In Handbook of Materials Modeling: Applications: Current and Emerging Materials; Andreoni, W., Yip, S., Eds.; Springer International Publishing: Cham, 2018; pp 1-21.

(72) Côté, A. S.; Cormack, A. N.; Tilocca, A. Reactive molecular dynamics: an effective tool for modelling the sol-gel synthesis of bioglasses. Journal of Materials Science 2017, 52, 9006-9013.

(73) Tuckerman, M. E.; Martyna, G. J. Understanding Modern Molecular Dynamics: Techniques and Applications. The Journal of Physical Chemistry B 2000, 104, $159-178$.

(74) Hospital, A.; Goñi, J. R.; Orozco, M.; Gelpí, J. L. Molecular dynamics simulations: advances and applications. Advances and applications in bioinformatics and chemistry 2015, 8, 37-47.

(75) Ferreiro-Rangel, C. A.; Gelb, L. D. Investigation of the Bulk Modulus of Silica Aerogel Using Molecular Dynamics Simulations of a Coarse-Grained Model. The Journal of Physical Chemistry B 2013, 117, 7095-7105, PMID: 23631801. 
(76) Brini, E.; Algaer, E. A.; Ganguly, P.; Li, C.; Rodríguez-Ropero, F.; van der Vegt, N. F. A. Systematic coarse-graining methods for soft matter simulations: a review. Soft Matter 2013, 9, 2108-2119.

(77) Merchant, B. A.; Madura, J. D. In Annual Reports in Computational Chemistry; Wheeler, R. A., Ed.; Annual Reports in Computational Chemistry; Elsevier, 2011; Vol. $7 ;$ pp $67-87$.

(78) Ingólfsson, H. I.; Lopez, C. A.; Uusitalo, J. J.; de Jong, D. H.; Gopal, S. M.; Periole, X.; Marrink, S. J. The power of coarse graining in biomolecular simulations. Wiley Interdisciplinary Reviews: Computational Molecular Science 2014, 4, 225248.

(79) Saunders, M. G.; Voth, G. A. Coarse-Graining Methods for Computational Biology. Annual Review of Biophysics 2013, 42, 73-93, PMID: 23451897.

(80) Gelb, L. D. Simulating Silica Aerogels with a Coarse-Grained Flexible Model and Langevin Dynamics. The Journal of Physical Chemistry C 2007, 111, 1579215802.

(81) Rahmani, A.; Benoit, C.; Poussigue, G. A fractal model for silica aerogels. Journal of Physics: Condensed Matter 1994, 6, 1483.

(82) Hasmy, A.; Anglaret, E.; Foret, M.; Pelous, J.; Jullien, R. Small-angle neutronscattering investigation of long-range correlations in silica aerogels: Simulations and experiments. Phys. Rev. B 1994, 50, 6006-6016.

(83) Anglaret, E.; Hasmy, A.; Jullien, R. Effect of container size on gelation time: experiments and simulations. Physical review letters 1995, 75, 4059.

(84) Bos, M. T. A.; van Opheusden, J. H. J. Brownian dynamics simulation of gelation and aging in interacting colloidal systems. Phys. Rev. E 1996, 53, 5044-5050.

(85) Rahmani, A.; Jund, P.; Benoit, C.; Jullien, R. Numerical study of the dynamic properties of silica aerogels. Journal of Physics: Condensed Matter 2001, 13, 5413. 
(86) Ma, H.-S.; Jullien, R.; Scherer, G. W. Dangling bond deflection model: Growth of gel network with loop structure. Phys. Rev. E 2002, 65, 041403.

(87) Liu, Q.; Lu, Z.; Zhu, M.; Yuan, Z.; Yang, Z.; Hu, Z.; Li, J. Simulation of the tensile properties of silica aerogels: the effects of cluster structure and primary particle size. Soft Matter 2014, 10, 6266-6277.

(88) Lu, Z.; Yuan, Z.; Liu, Q.; Hu, Z.; Xie, F.; Zhu, M. Multi-scale simulation of the tensile properties of fiber-reinforced silica aerogel composites. Materials Science and Engineering: A 2015, 625, $278-287$.

(89) Ferreiro-Rangel, C. A.; Gelb, L. D. Computational Study of Uniaxial Deformations in Silica Aerogel Using a Coarse-Grained Model. The Journal of Physical Chemistry B 2015, 119, 8640-8650, PMID: 26039801.

(90) Elanany, M.; Selvam, P.; Yokosuka, T.; Takami, S.; Kubo, M.; Imamura, A.; Miyamoto, A. A Quantum Molecular Dynamics Simulation Study of the Initial Hydrolysis Step in Sol-Gel Process. The Journal of Physical Chemistry B 2003, 107, $1518-1524$.

(91) Trinh, T. T.; Jansen, A. P. J.; van Santen, R. A. Mechanism of Oligomerization Reactions of Silica. The Journal of Physical Chemistry B 2006, 110, 23099-23106, PMID: 17107150.

(92) Pereira, J. C. G.; R. A. Catlow, C.; D. Price, G. Silica condensation reaction: an ab initio study. Chem. Commun. 1998, 1387-1388.

(93) Trinh, T. T.; Jansen, A. P. J.; van Santen, R. A.; Meijer, E. J. Role of Water in Silica Oligomerization. The Journal of Physical Chemistry C 2009, 113, 26472652 .

(94) Pereira, J. C. G.; Catlow, C. R. A.; Price, G. D. Ab Initio Studies of Silica-Based Clusters. Part I. Energies and Conformations of Simple Clusters. The Journal of Physical Chemistry A 1999, 103, 3252-3267. 
(95) Pereira, J. C. G.; Catlow, C. R. A.; Price, G. D. Ab Initio Studies of Silica-Based Clusters. Part II. Structures and Energies of Complex Clusters. The Journal of Physical Chemistry A 1999, 103, 3268-3284.

(96) Zhang, X.-Q.; Trinh, T. T.; van Santen, R. A.; Jansen, A. P. J. Mechanism of the Initial Stage of Silicate Oligomerization. Journal of the American Chemical Society 2011, 133, 6613-6625, PMID: 21486018.

(97) Monsivais-Gámez, E.; Ruiz, F.; Martínez, J. R. Four-membered rings family in the Si-O extended rocking IR band from quantum chemistry calculations. Journal of Sol-Gel Science and Technology 2007, 43, 65-72.

(98) Ospino, I.; Luquin, A.; Jiménez-Ruiz, M.; Pérez-Landazábal, J. I.; Recarte, V.; Echeverría, J. C.; Laguna, M.; Urtasun, A. A.; Garrido, J. J. Computational Modeling and Inelastic Neutron Scattering Contributions to the Study of Methyl-silica Xerogels: A Combined Theoretical and Experimental Analysis. The Journal of Physical Chemistry C 2017, 121, 22836-22845.

(99) Bennett, M. D.; Wolters, C. J.; Brandstadt, K. F.; Tecklenburg, M. M. Raman spectroscopy and DFT calculations of intermediates in the hydrolysis of methylmethoxysilanes. Journal of Molecular Structure 2012, 1023, $204-211$.

(100) Borba, A.; Almangano, M.; Portugal, A. A.; Patrício, R.; Simões, P. N. Methylsilsesquioxane-Based Aerogel Systems - Insights into the Role of the Formation of Molecular Clusters. The Journal of Physical Chemistry A 2016, 120, 4079-4088, PMID: 27213224.

(101) Maximiano, P.; Durães, L.; Simões, P. N. Organically-modified silica aerogels: A density functional theory study. The Journal of Supercritical Fluids 2019, 147, 138 $-148$.

(102) Woignier, T.; Phalippou, J.; Vacher, R.; Pelous, J.; Courtens, E. Different kinds of fractal structures in silica aerogels. Journal of Non-Crystalline Solids 1990, 121, 198 - 201, Proceedings of the Fifth International Workshop on Glasses and Ceramics from Gels. 
(103) Chevallier, T.; Woignier, T.; Toucet, J.; Blanchart, E.; Dieudonné, P. Fractal structure in natural gels: effect on carbon sequestration in volcanic soils. Journal of Sol-Gel Science and Technology 2008, 48, 231-238.

(104) Beckers, J.; de Leeuw, S. Molecular dynamics simulation of nanoporous silica. Journal of Non-Crystalline Solids 2000, 261, 87 - 100.

(105) Nakano, A.; Bi, L.; Kalia, R. K.; Vashishta, P. Molecular-dynamics study of the structural correlation of porous silica with use of a parallel computer. Phys. Rev. $B$ 1994, 49, 9441-9452.

(106) Kieffer, J.; Angell, C. Generation of fractal structures by negative pressure rupturing of $\mathrm{SiO}_{2}$ glass. Journal of Non-Crystalline Solids 1988, 106, 336 - 342 .

(107) Pohl, P. I.; Faulon, J.-L.; Smith, D. M. Molecular dynamics computer simulations of silica aerogels. Journal of Non-Crystalline Solids 1995, 186, 349-355, Proceedings of the Fourth International Symposium on AEROGELS.

(108) Lei, J.; Liu, Z.; Yeo, J.; Ng, T. Y. Determination of the Young's modulus of silica aerogels - an analytical-numerical approach. Soft Matter 2013, 9, 11367-11373.

(109) Einarsrud, M.-A.; Haereid, S. Preparation of transparent, monolithic silica xerogels with low density. Journal of Sol-Gel Science and Technology 1994, 2, 903-906.

(110) Vareda, J. P.; Maximiano, P.; Cunha, L. P.; Ferreira, A. F.; Simões, P. N.; Durães, L. Effect of different types of surfactants on the microstructure of methyltrimethoxysilane-derived silica aerogels: A combined experimental and computational approach. Journal of Colloid and Interface Science 2018, 512, 64-76.

(111) Klein, L. C. Sol-Gel Processing of Silicates. Annual Review of Materials Science 1985, 15, 227-248.

(112) Pouxviel, J.; Boilot, J.; Beloeil, J.; Lallemand, J. NMR study of the sol/gel polymerization. Journal of Non-Crystalline Solids 1987, 89, 345 - 360. 
(113) Garofalini, S. H.; Martin, G. Molecular Simulations of the Polymerization of Silicic Acid Molecules and Network Formation. The Journal of Physical Chemistry 1994, 98, 1311-1316.

(114) Rao, N. Z.; Gelb, L. D. Molecular Dynamics Simulations of the Polymerization of Aqueous Silicic Acid and Analysis of the Effects of Concentration on Silica Polymorph Distributions, Growth Mechanisms, and Reaction Kinetics. The Journal of Physical Chemistry B 2004, 108, 12418-12428.

(115) Bhattacharya, S.; Kieffer, J. Molecular Dynamics Simulation Study of Growth Regimes during polycondensation of Silicic Acid: from Silica Nanoparticles to Porous Gels. The Journal of Physical Chemistry C 2008, 112, 1764-1771.

(116) Woignier, T.; Pelous, J.; Phalippou, J.; Vacher, R.; Courtens, E. Elastic properties of silica aerogels. Journal of Non-Crystalline Solids 1987, 95-96, 1197 - 1202.

(117) Woignier, T.; Reynes, J.; Alaoui, A. H.; Beurroies, I.; Phalippou, J. Different kinds of structure in aerogels: relationships with the mechanical properties. Journal of Non-Crystalline Solids 1998, 241, $45-52$.

(118) Campbell, T.; Kalia, R. K.; Nakano, A.; Shimojo, F.; Tsuruta, K.; Vashishta, P.; Ogata, S. Structural Correlations and Mechanical Behavior in Nanophase Silica Glasses. Phys. Rev. Lett. 1999, 82, 4018-4021.

(119) Groß, J.; Fricke, J. Scaling of elastic properties in highly porous nanostructured aerogels. Nanostructured Materials 1995, 6, 905 - 908, Proceedings of the Second International Conference on Nanostructured Materials.

(120) Hansen, J. S.; Schröder, T. B.; Dyre, J. C. Simplistic Coulomb Forces in Molecular Dynamics: Comparing the Wolf and Shifted-Force Approximations. The Journal of Physical Chemistry B 2012, 116, 5738-5743, PMID: 22497264.

(121) Patil, S. P.; Shendye, P.; Markert, B. Molecular dynamics investigation of the shock response of silica aerogels. Materialia 2019, 6, 100315. 
(122) Patil, S. P.; Parale, V. G.; Park, H.-H.; Markert, B. Molecular dynamics and experimental studies of nanoindentation on nanoporous silica aerogels. Materials Science and Engineering: A 2019, 742, 344-352.

(123) Patil, S. P. Nanoindentation of Graphene-Reinforced Silica Aerogel: A Molecular Dynamics Study. Molecules 2019, 24.

(124) Morthomas, J.; Gonçalves, W.; Perez, M.; Foray, G.; Martin, C. L.; Chantrenne, P. A novel method to predict the thermal conductivity of nanoporous materials from atomistic simulations. Journal of Non-Crystalline Solids 2019, 516, 89 - 98.

(125) Brinker, C.; Brow, R.; Tallant, D.; Kirkpatrick, R. Surface structure and chemistry of high surface area silica gels. Journal of Non-Crystalline Solids 1990, 120, 26 33.

(126) Chuang, I.-S.; Maciel, G. E. A Detailed Model of Local Structure and Silanol Hydrogen Bonding of Silica Gel Surfaces. The Journal of Physical Chemistry B 1997, 101, 3052-3064.

(127) Zhuravlev, L. T. Concentration of hydroxyl groups on the surface of amorphous silicas. Langmuir 1987, 3, 316-318.

(128) Zhuravlev, L. The surface chemistry of amorphous silica. Zhuravlev model. Colloids and Surfaces A: Physicochemical and Engineering Aspects 2000, 173, 1 - 38.

(129) Ceresoli, D.; Bernasconi, M.; Iarlori, S.; Parrinello, M.; Tosatti, E. Two-Membered Silicon Rings on the Dehydroxylated Surface of Silica. Phys. Rev. Lett. 2000, 84, 3887-3890.

(130) Lockwood, G. K.; Garofalini, S. H. Proton Dynamics at the Water-Silica Interface via Dissociative Molecular Dynamics. The Journal of Physical Chemistry C 2014, 118, 29750-29759.

(131) Mahadevan, T. S.; Garofalini, S. H. Dissociative Chemisorption of Water onto Silica Surfaces and Formation of Hydronium Ions. The Journal of Physical Chemistry C 2008, 112, 1507-1515. 
(132) Redmill, P. S.; McCabe, C. Molecular Dynamics Study of the Behavior of Selected Nanoscale Building Blocks in a Gel-Phase Lipid Bilayer. The Journal of Physical Chemistry B 2010, 114, 9165-9172, PMID: 20583770.

(133) Albers, P.; Maier, M.; Reisinger, M.; Hannebauer, B.; Weinand, R. Physical boundaries within aggregates: differences between amorphous, para-crystalline, and crystalline Structures. Crystal Research and Technology 2015, 50, 846-865.

(134) Paek, E.; Hwang, G. S. A computational analysis of graphene adhesion on amorphous silica. Journal of Applied Physics 2013, 113, 164901.

(135) Gao, W.; Xiao, P.; Henkelman, G.; Liechti, K. M.; Huang, R. Interfacial adhesion between graphene and silicon dioxide by density functional theory with van der Waals corrections. Journal of Physics D: Applied Physics 2014, 47, 255301.

(136) de Lara, L. S.; Michelon, M. F.; Metin, C. O.; Nguyen, Q. P.; Miranda, C. R. Interface tension of silica hydroxylated nanoparticle with brine: A combined experimental and molecular dynamics study. The Journal of Chemical Physics 2012, $136,164702$.

(137) Nejad, M. A.; Urbassek, H. M. Insulin adsorption on functionalized silica surfaces: an accelerated molecular dynamics study. Journal of Molecular Modeling 2018, $24,89$.

(138) Khanniche, S.; Mathieu, D.; Pereira, F.; Hairault, L. Atomistic models of hydroxylated, ethoxylated and methylated silica surfaces and nitrogen adsorption isotherms: A molecular dynamics approach. Microporous and Mesoporous Materials 2017, 250, $158-169$.

(139) Brunk, E.; Rothlisberger, U. Mixed Quantum Mechanical/Molecular Mechanical Molecular Dynamics Simulations of Biological Systems in Ground and Electronically Excited States. Chemical Reviews 2015, 115, 6217-6263. 